\title{
Dietary Fat and Coronary Heart Disease: Summary of Evidence from Prospective Cohort and Randomised Controlled Trials
}

\author{
C. Murray Skeaff Jody Miller \\ Department of Human Nutrition, University of Otago, Dunedin, New Zealand
}

\section{Introduction}

This article summarises the evidence from cohort studies and randomised controlled trials for the importance of total fat and dietary fatty acids for risk of coronary heart disease (CHD). Its purpose is to assist the expert consultation group to make evidence-based recommendations about fat, fatty acids and human health.

Ecological studies that compare differences in CHD rates between mean intakes of fatty acids in different populations are uniquely informative, as such associations are virtually unaffected by regression dilution bias. The best known ecological study of diet and CHD is the Seven Countries Study, which consisted of 16 cohorts in 7 different countries involving a total of 12,763 middleaged men that were examined between 1958 and 1964 [Keys, 1980]. The Seven Countries Study showed that death rates from CHD during 10 and 15 years of followup across the 16 cohorts were positively associated with dietary intake of saturated fat (SFA) at baseline and inversely associated with dietary intake of monounsaturated fat (MUFA) [Keys et al., 1986]. The results showed that a substantial proportion of the variation in CHD death rates between geographical regions was explained by differences in intake of SFA and MUFA fat. At 25 years of follow-up only the association with baseline SFA intake remained [Kromhout et al., 1995b]. Moreover, the Seven
Countries Study also demonstrated strong associations between mean intakes of SFA and mean levels of serum total cholesterol [Keys, 1980]. The study prompted the 'diet heart' hypothesis that high intakes of SFA and cholesterol and low intakes of polyunsaturated fats (PUFA) increase the level of total cholesterol and ultimately result in the development of CHD. Indeed, the early results of the Seven Countries Study prompted an explosion of epidemiological, clinical, and basic research into the role of dietary fat in CHD.

The results of dietary feeding trials (or 'metabolic ward' studies) which measured blood lipids in healthy volunteers after administration of controlled diets with varying intakes of fats were concordant with the findings of the associations observed between intakes of different fatty acids and changes in blood cholesterol levels observed in the ecological studies. In particular, Keys et al. [1965] and Hegsted et al. [1965] demonstrated that average change in serum cholesterol concentrations could be predicted as equations for the changes in intake of SFA and PUFA and dietary cholesterol. The concordance of the results of the ecological and the metabolic ward studies probably relate to the limited amount of measurement error in both study designs. In view of these findings, some investigators have concluded that use of cholesterol as an intermediary factor is the most rational way of studying the associations between dietary fat and CHD,

\section{KARGER \\ Fax +4161306 1234 \\ E-Mail karger@karger.ch}

www.karger.com
(C) 2009 S. Karger AG, Basel and FAO

0250-6807/09/0553-0173\$26.00/0

Accessible online at:

www.karger.com/anm
C. Murray Skeaff

Department of Human Nutrition, University of Otago

PO Box 56

Dunedin (New Zealand)

Tel. +64 34797 688, Fax +64 34797 958, E-Mail murray.skeaff@otago.ac.nz 
with appropriate correction for measurement error in both study designs. Nevertheless, many investigators have examined the associations of differences in intake of fatty acids directly with CHD risk within populations. The present review summarises the evidence from the cohort studies and dietary intervention trials that examined the effects of differences in diet (or exchanges of particular fats by another or by carbohydrate) on risk of CHD.

In addition to the evidence of the importance of reducing the intake of SFA and dietary cholesterol for prevention of CHD, other sources of evidence have focussed on finding the best replacement for SFA and the relative roles of n-3 and n-6 PUFAs. Based on observations in the mid 1970 s of the Greenland Inuit and subsequently in clinical trials, Bang and Dyerberg [Bang et al., 1976; Dyerberg et al., 1978; Dyerberg and Bang, 1979], showed that n-3 long chain polyunsaturated fatty acids (LCPUFA) might have cardio-protective effects independently of their effects on serum cholesterol concentrations.

The articles by Sanders (pp 162-172 of this issue) and Galli and Calder (pp 123-139 of this issue) respectively examine the effects of dietary fats on blood lipids and other biomarkers of inflammation and other factors that may affect CHD risk. The purpose of this article is to summarise the evidence from cohort studies and randomised controlled trials of the relation between dietary fat and risk of CHD.

\section{Methods}

Cohort studies and controlled trials of dietary fat and CHD mortality or morbidity were identified by searching the Cochrane Library and examining Cochrane reviews [Hooper et al., 2001, 2004a]; by keyword searches of article databases using Medline, Embase, SCOPUS, Web of Science and PubMed; by examining the tables, figures and list of references in review articles [Hooper et al., 2004b; Mozaffarian et al., 2006; Booker and Mann, 2008; Erkkila et al., 2008], systematic reviews [Wang et al., 2006], metaanalyses [Bucher et al., 2002; Brouwer et al., 2004; He et al., 2004; Whelton et al., 2004; Yzebe and Lievre, 2004; Mozaffarian and Rimm, 2006; Jenkins et al., 2008] and original articles; and by searching for papers that had cited relevant cohort and intervention studies. The present review was limited to English language publications.

Information about study design, methods and key results were extracted from the original source or, in a few instances when the original source was unobtainable, from peer-reviewed articles that had cited the original study results. The cohort study endpoints reviewed were CHD death, CHD events, and non-fatal CHD. The randomised clinical trial endpoints included total mortality. For the n-3 LCPUFA/Fish trials we also examined restenosis/occlusion/revascularization, non-fatal myocardial infarction, and angina.
To summarise the results from published cohort studies, random effects meta-analysis was used to calculate summary estimates of the relative risk (RR) of CHD in high compared with low exposure to dietary fat or its components: trans fatty acids (TFA), SFA, MUFA, PUFA, and n-3 LCPUFA. Multiple variable adjusted RRs were extracted from the original sources and used, when available. Table 1 summarises the covariates that were included in the multivariable analysis for each cohort study.

Studies in which dietary fat exposure was assessed using fatty acid biomarkers were included in the meta-analyses of high compared with low fat exposure alongside studies in which fat intake was assessed using traditional methods of dietary assessment. Thus, for example, in the meta-analysis of cohort studies of n-3 LCPUFA and risk of CHD we included studies of dietary fish, fish oil or n-3 LCPUFA intake as well as studies in which exposure was assessed using fatty acid biomarkers. For MUFA we only included studies in which exposure was determined by dietary assessment because blood fatty acids are not good biomarkers of MUFA intake. The dietary assessment methods used in the cohort studies included single 24-hour recall, diet records, diet histories and food frequency questionnaires collected at baseline or from the same participants at various times throughout follow-up (table 2).

Many studies reported the RR of CHD for an incremental change in fat intake. Units of incremental change included $2 \%$ or $5 \%$ of energy, 1 standard deviation and $100 \mathrm{~g}$ of fat. In most studies where the RR associated with an incremental increase in percent energy from fat type was reported, the statistical analysis was adjusted for other types of fat (SFA, MUFA, PUFA and TFA) so that the result represents the $\mathrm{RR}$ associated with replacing carbohydrate with the specific type of fat. We included in the results a forest plot of the RRs of CHD for any incremental change, but suppressed the estimate of overall risk because the unit of comparison was markedly different between studies. Separate metaanalyses were performed to generate summary estimates of risk for $2 \%$ energy increments for TFA and 5\% energy increments for SFA, MUFA and PUFA.

To avoid duplication of data from individual studies that provided multiple reports, reports with the longest duration of follow-up were selected for review. For n-3 LCPUFA and CHD cohort studies we included in the meta-analysis only the risk associated with the n-3 LCPUFA biomarkers in the first instance, or fish consumption if no biomarker was measured.

Cohort studies that did not report a RR associated with intake of dietary fats were excluded from the meta-analyses. The most common alternate measure of association between dietary fat and disease was a test for differences in dietary fat intake or level of fatty acid biomarkers between participants who did or did not develop CHD during follow-up. In all cases the differences were not multivariable-adjusted comparisons and therefore subject to potential confounding; accordingly we have presented the results in the supplementary tables but excluded them from this review. Supplementary materials for this article are available online at www.karger.com/doi/10.1159/000229002.

In the meta-analyses of results from randomised controlled trials of dietary fat and CHD we classified the studies into 4 general categories according to the primary goal of the dietary treatment: (1) diets involving a change in the polyunsaturated to saturated fat $(\mathrm{P} / \mathrm{S})$ ratio of the diet, with or without a reduction in total fat intake; (2) diets involving a reduction in total fat; (3) diets 
involving an increase in fish or fish oil intake, and (4) diets involving an increase in foods rich in $\alpha$-linolenic acid. A few studies could not be grouped into these categories and were excluded from the meta-analyses but are reported in the online supplementary tables. Trials that involved multi-factorial interventions (e.g. MRFIT) were excluded from the meta-analysis. Information about the number of participants in the treatment and control groups with or without a coronary disease endpoint during follow-up were extracted from the published trial results. As a sensitivity analysis, trials in which the $\mathrm{P} / \mathrm{S}$ diet produced a reduction in serum cholesterol relative to the control group were identified and examined separately as a measure of compliance. A separate meta-analysis was performed for trials in which participant compliance with dietary treatment resulted in a reduction in serum cholesterol.

For the meta-analysis of randomised controlled trials of $n-3$ LCPUFA and CHD risk, we included any trial in which the intervention involved increased consumption of fish, fish oil or an n-3 LCPUFA purified oil.

All the RRs were displayed graphically as Forrest plots with a weighting inversely proportional to the variance of each study or trial. Summary estimates of risk and $95 \%$ CI were estimated by means of random effects meta-analysis used in Stata version 10 (Stata Corp., College Station, Tex., USA).

\section{Results}

\section{Update of Previous Meta-Analyses}

The present meta-analysis excludes 3 trials that were included in the review of dietary fat modification and CHD by Hooper et al. [2001]: the olive oil arm of the study by Rose et al. [1965] was excluded as it did not fit within the low-fat or PUFA-SFA intervention criteria, and the Sydney-diet [Blacket et al., 1979] and Veterans' Diet and Skin Cancer [Black et al., 1994] studies were excluded as they reported only cardiovascular disease and not CHD endpoints. We included 2 additional trials: the Finnish Mental Hospital [Turpeinen, 1979; Miettinen et al., 1983] and the Women's Health Initiative [Howard et al., 2006].

For the fish or n-3 LCPUFA trials, we excluded 3 studies that were included in the meta-analysis by Hooper et al. [2006], as they investigated $\alpha$-linolenic supplementation rather than n-3 LCPUFA [Borchgrevink et al., 1966; Natvig et al., 1968; Bemelmans et al., 2002] and we excluded 1 trial with methodological concerns [Singh et al., 1997]. Five additional trials were included in the present meta-analysis [Leaf et al., 2005; Raitt et al., 2005; Brouwer et al., 2006; Yokoyama et al., 2007; GISSI-HF Investigators, 2008].

The present meta-analysis updates the review of fish intake and CHD by Mozaffarian and Rimm [2006] with the inclusion of 2 additional trials [Yokoyama et al., 2007;
GISSI-HF Investigators, 2008] and 6 cohorts [Norell et al., 1986; Mann et al., 1997; Pietinen et al., 1997; Rissanen et al., 2000; Erkkila et al., 2003; Streppel et al., 2008]. We excluded 1 study that was included in Mozaffarian and Rimm's review [Kromhout et al., 1985] as a report for a longer duration was available [Streppel et al., 2008] and 1 trial with methodological concerns [Singh et al., 1997].

\section{Cohort Studies of Dietary Fat and CHD}

Selected characteristics of the 28 individual cohort studies are shown in online supplementary table 1. A few studies (e.g. Nurses' Health Study) have been duplicated because reports from the same study have been published at periodic years of follow-up. Data includes the geographical location, start year, duration of follow-up, number of participants, participant exclusion criteria, method of assessing dietary exposure, type of CHD event assessed, overall event rate, and the method of determining the association between fat exposure and CHD risk; for example, RR of disease in high compared with low consumers.

There were about 6,600 CHD deaths amongst the 280,000 participants in the cohort studies during approximately 3.7 million person-years of follow-up. CHD mortality rates ranged from 45 to 2,300 deaths per 100,000 person-years. The duration of follow-up varied from 4 to 25 years. With few exceptions, the studies were conducted in North America and in Europe. Nineteen of the 28 cohorts included only men, accounting for 1.84 million person-years of follow-up; the Nurses' Health Study was limited to women and accounted for more than $80 \%$ of person-years of follow-up amongst women in all cohorts. The age at recruitment varied from 40 to 65 years.

\section{Meta-Analysis of Cohort Studies of Total Fat and CHD}

Intake of total fat was not significantly associated with CHD mortality, with a RR for highest compared with the lowest category of 0.94 (95\% CI $0.74-1.18, \mathrm{p}=0.583$; fig. 1 ). Intake of total fat was also unrelated to CHD events (RR $0.93,95 \%$ CI $0.84-1.03, \mathrm{p}=0.177)$. For the analysis that used $5 \%$ percent increase in total fat intake, there was no significant association of total fat intake with CHD mortality (RR 1.06, 95\% CI $0.88-1.28, \mathrm{p}=0.517$ ) or $\mathrm{CHD}$ events (RR 1.02, 95\% CI 0.98-1.05, p = 0.404) per 5\% total energy (TE) increment in total fat intake (fig. 3). The range of total fat intake (mean or median) varied from 23 to $30 \% \mathrm{TE}$ in the lowest category to 38 to $47 \% \mathrm{TE}$ in the highest category (table 3 ). Overall, the mean or median total fat intake in all cohort studies varied from 27 to $47 \%$ TE (online suppl. table 2). 


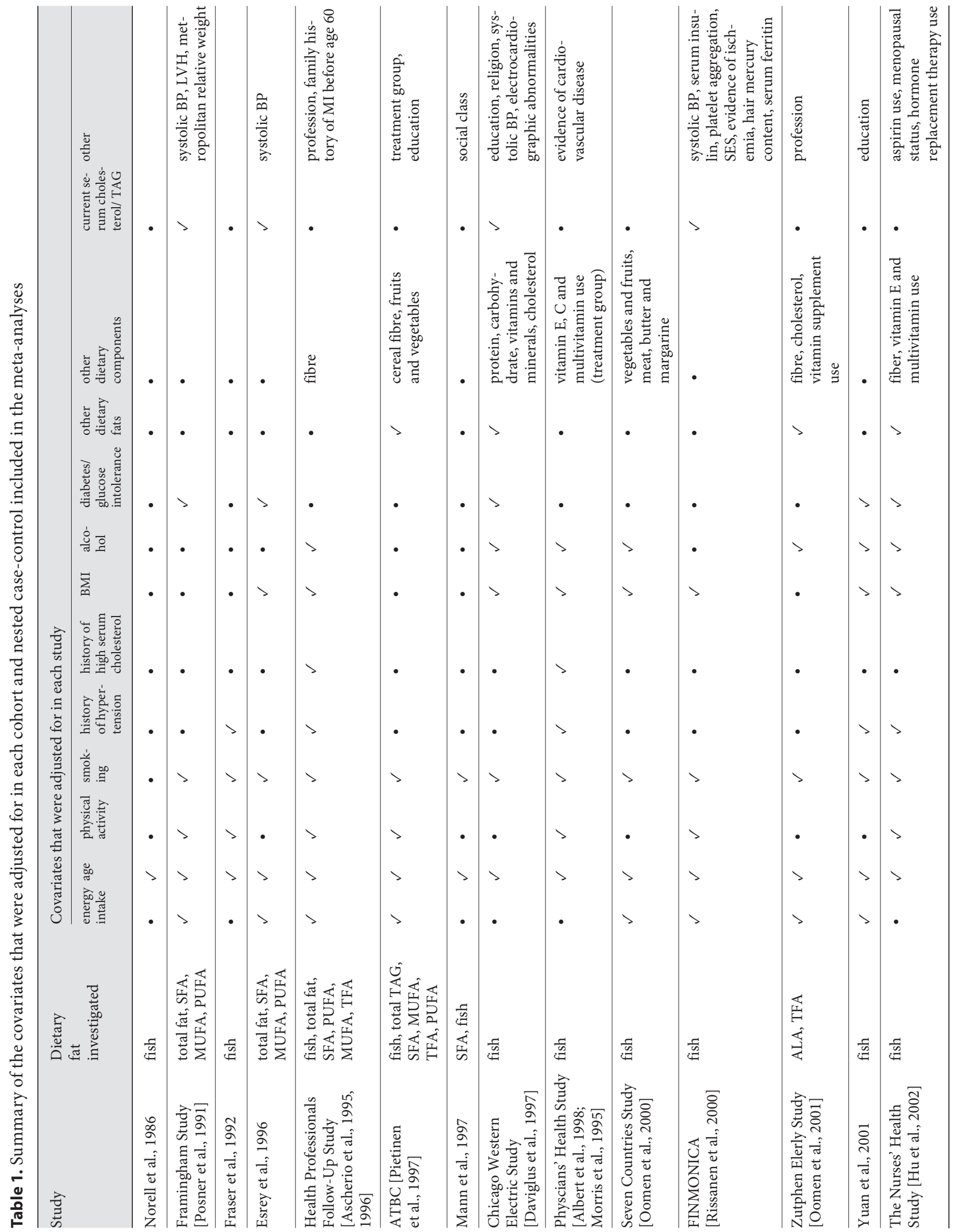




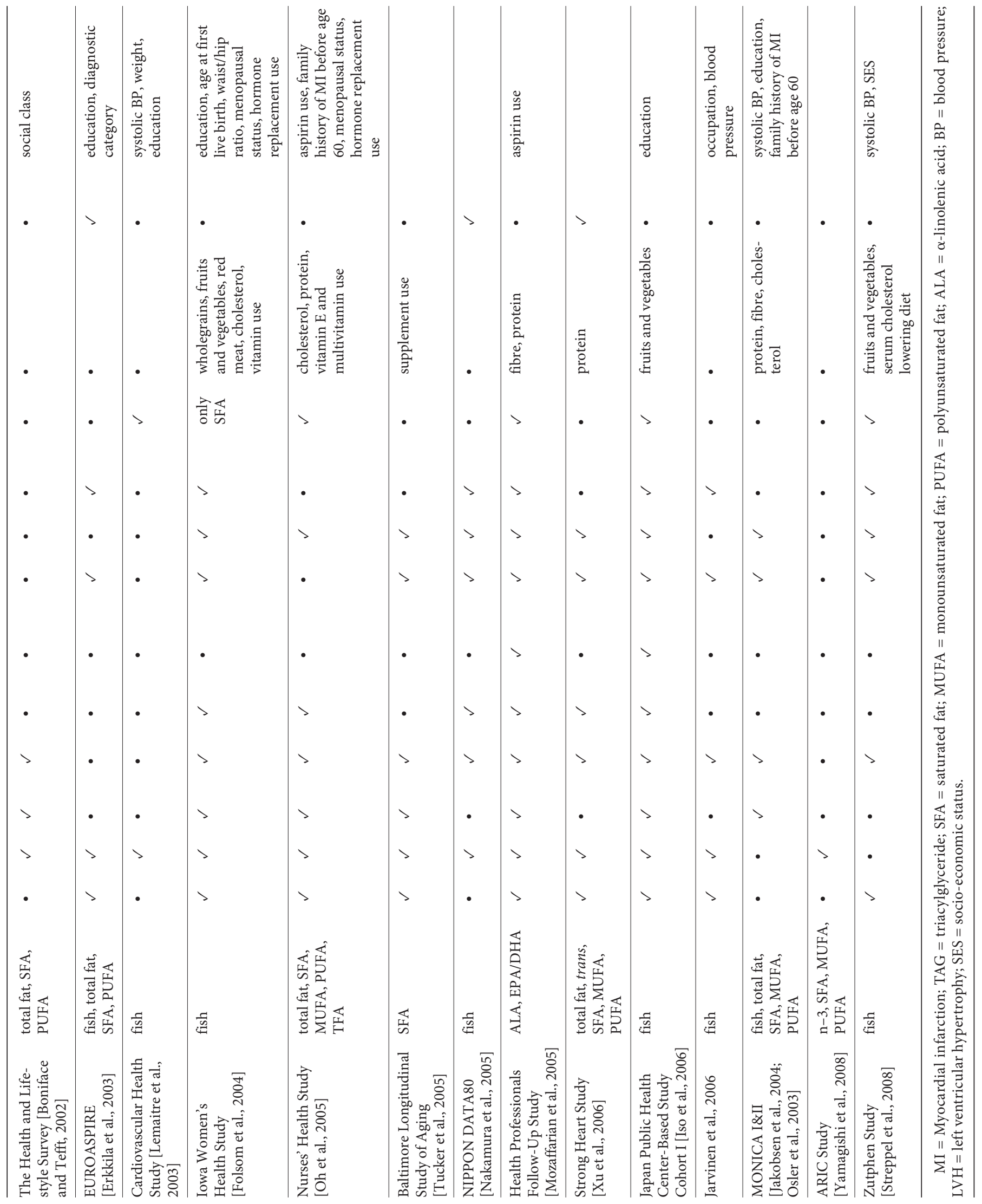


Table 2. Dietary assessment methods used for cohort studies included in the meta-analyses

\begin{tabular}{|c|c|c|}
\hline Study & Diet assessment method & Dietary assessment completed \\
\hline Norell et al., 1986 & food frequency questionnaire & baseline \\
\hline Framingham Study [Posner et al., 1991] & 24-hour recall & baseline \\
\hline Fraser et al., 1992 & food frequency questionnaire & baseline \\
\hline Esrey et al., 1996 & 24-hour recall & baseline \\
\hline Health Professionals Follow-Up Study [Ascherio et al., 1995, 1996] & food frequency questionnaire & baseline \\
\hline ATBC Study [Pietinen et al., 1997] & food frequency questionnaire & baseline \\
\hline Mann et al., 1997 & food frequency questionnaire & baseline \\
\hline Physicians' Health Study [Morris et al., 1995; Albert et al., 2002] & food frequency questionnaire & baseline and 1 year later \\
\hline Seven Countries Study [Kromhout et al., 1995b] & weighed diet records & baseline \\
\hline Kromhout et al., 1995a (Rotterdam) & cross-check dietary history & baseline \\
\hline Chicago Western Electric Study [Daviglus et al., 1997] & dietary history & baseline and 1 year later \\
\hline Yuan et al., 2001 & food frequency questionnaire & baseline \\
\hline Seven Countries Study [Oomen et al., 2000] & dietary history and food frequency checklist & baseline and 1 year later \\
\hline Health and Lifestyle Survey [Boniface and Tefft 2002] & food frequency questionnaire & baseline \\
\hline Cardiovascular Health Study [Mozaffarian et al., 2003] & food frequency questionnaire & baseline \\
\hline EUROASPIRE Study [Erkkila et al., 2003] & 4-day estimated food record & baseline \\
\hline MONICA I\&II [Osler et al., 2003; Jakobsen et al., 2004] & 7-day weighed diet record & baseline \\
\hline Iowa Women's Health Study [Folsom and Demissie, 2004] & food frequency questionnaire & baseline \\
\hline NIPPON DATA80 [Nakamura et al., 2005] & food frequency questionnaire & baseline \\
\hline Nurses' Health Study [Oh et al., 2005] & food frequency questionnaire & collected 1980, 1984, 1986, 1990, 1994 \\
\hline Baltimore Longitudinal Study of Aging [Tucker et al., 2005] & 7 -day diet records & 4 times throughout follow-up \\
\hline Health Professionals Follow-Up Study [Mozaffarian et al., 2005] & food frequency questionnaire & baseline and every 4 years \\
\hline Strong Heart Study [Xu et al., 2006] & 24-hour diet recall & 4 years after start of study \\
\hline Jarvinen et al., 2006 & dietary history & baseline \\
\hline Japan Public Health Center-Based Study Cohort 1 [Iso et al., 2006] & food frequency questionnaire & baseline and 5 years later \\
\hline Zutphen Study [Streppel et al., 2008] & dietary history & baseline \\
\hline
\end{tabular}

Table 3. Summary estimates of relative risk from random effects meta-analysis of prospective cohort study

\begin{tabular}{|c|c|c|c|c|c|c|}
\hline \multirow[t]{2}{*}{ Fat } & \multicolumn{4}{|c|}{ Relative risk (95\% CI) } & \multicolumn{2}{|c|}{$\begin{array}{l}\text { Range of mean fat intake in low and } \\
\text { high categories across cohorts, \% TE }\end{array}$} \\
\hline & CHD death & $\mathrm{p}$ value & CHD events & $\mathrm{p}$ value & low & high \\
\hline \multicolumn{7}{|c|}{ High compared with low intake } \\
\hline Total fat & $0.94(0.74-1.18)$ & 0.583 & $0.93(0.84-1.03)$ & 0.177 & $23-30$ & $38-47$ \\
\hline TFA & $1.32(1.08-1.61)$ & 0.006 & $1.25(1.07-1.46)$ & 0.007 & $0.8-2.4$ & $1.6-6.4$ \\
\hline PUFA & $1.25(1.06-1.47)$ & 0.009 & $0.97(0.74-1.27)$ & 0.825 & $3-4$ & $6-10$ \\
\hline \multirow[t]{2}{*}{$n-3$ LCPUFA $^{a}$} & $0.82(0.71-0.94)$ & 0.006 & $0.87(0.71-1.10)$ & 0.066 & $0-0.3 \mathrm{~g} / \mathrm{day}^{\mathrm{b}}$ & $0.37-2.5 \mathrm{~g} / \mathrm{day}^{\mathrm{b}}$ \\
\hline & & & & & $0-23 \mathrm{~g} / \mathrm{day}^{\mathrm{c}}$ & $22-180 \mathrm{~g} /$ day $^{\mathrm{c}}$ \\
\hline \multicolumn{7}{|c|}{ Per \% TE increment } \\
\hline Total fat (5\% TE) & $1.06(0.88-1.28)$ & 0.517 & $1.02(0.98-1.05)$ & 0.404 & & \\
\hline
\end{tabular}

${ }^{a}$ Includes trials of fish consumption, n-3 LCPUFA intake, and biomarkers. ${ }^{\mathrm{b}}$ Grams of n-3 LCPUFA per day. ${ }^{\mathrm{c}}$ Grams of fish per day. 


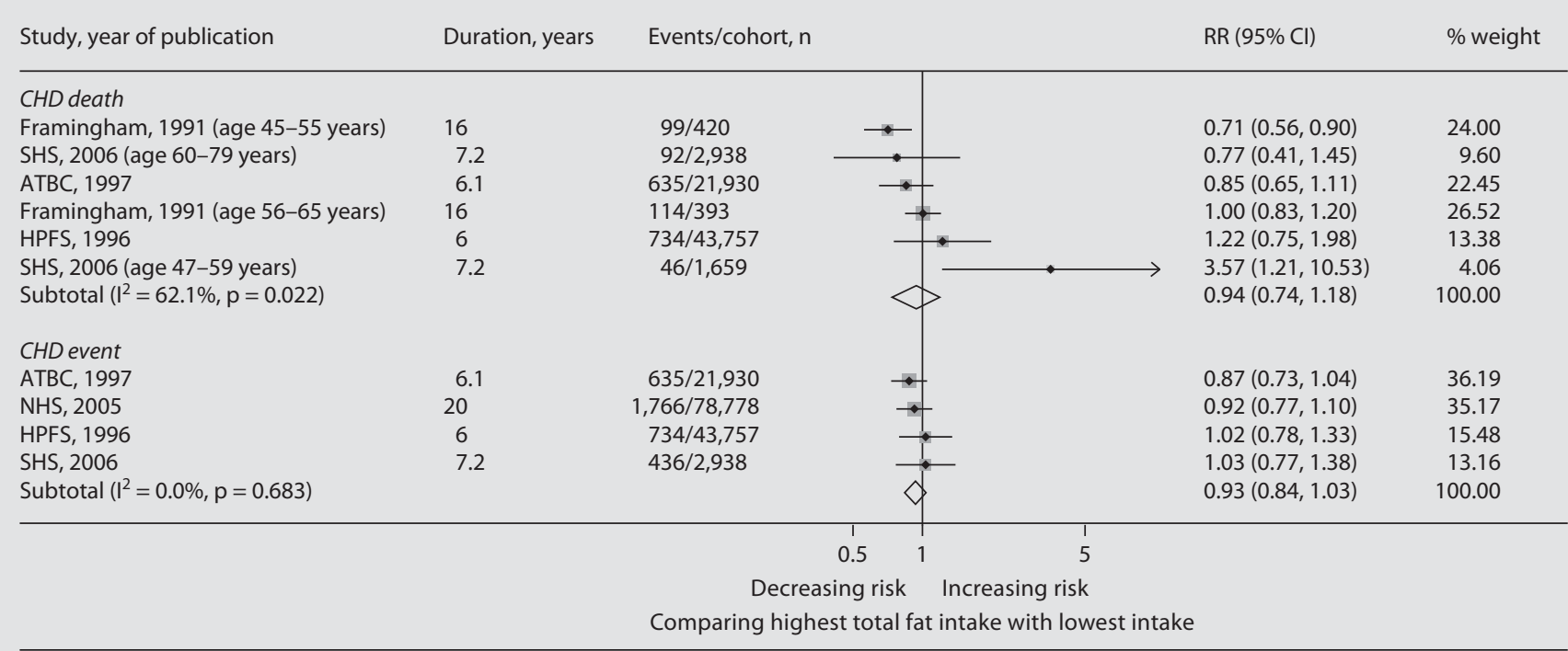

$p=0.583$ for CHD death subtotal; $p=0.177$ for CHD event subtotal. The relative risks correspond to comparisons of the highest total fat intakes with the lowest intakes, except the Framingham Study, which compared the sample mean fat intakes with the National Cholesterol Education Program Recommendations. The relative risks are the fully adjusted, multivariate results for each study.

Framingham = Framingham Study [Posner et al., 1991]; SHS = Strong Heart Study [Xu et al., 2006]; ATBC = Alpha-Tocopherol, Beta-Carotene Cancer Prevention Study [Pietinen et al., 1997]; HPFS = Health Professionals Follow-Up Study [Ascherio et al., 1996]; NHS = Nurses' Health Study [Oh et al., 2005]. Refer to online suppl. tables 1 and 3 for full study details.

Fig. 1. Meta-analysis of total fat intake and CHD; prospective cohorts.

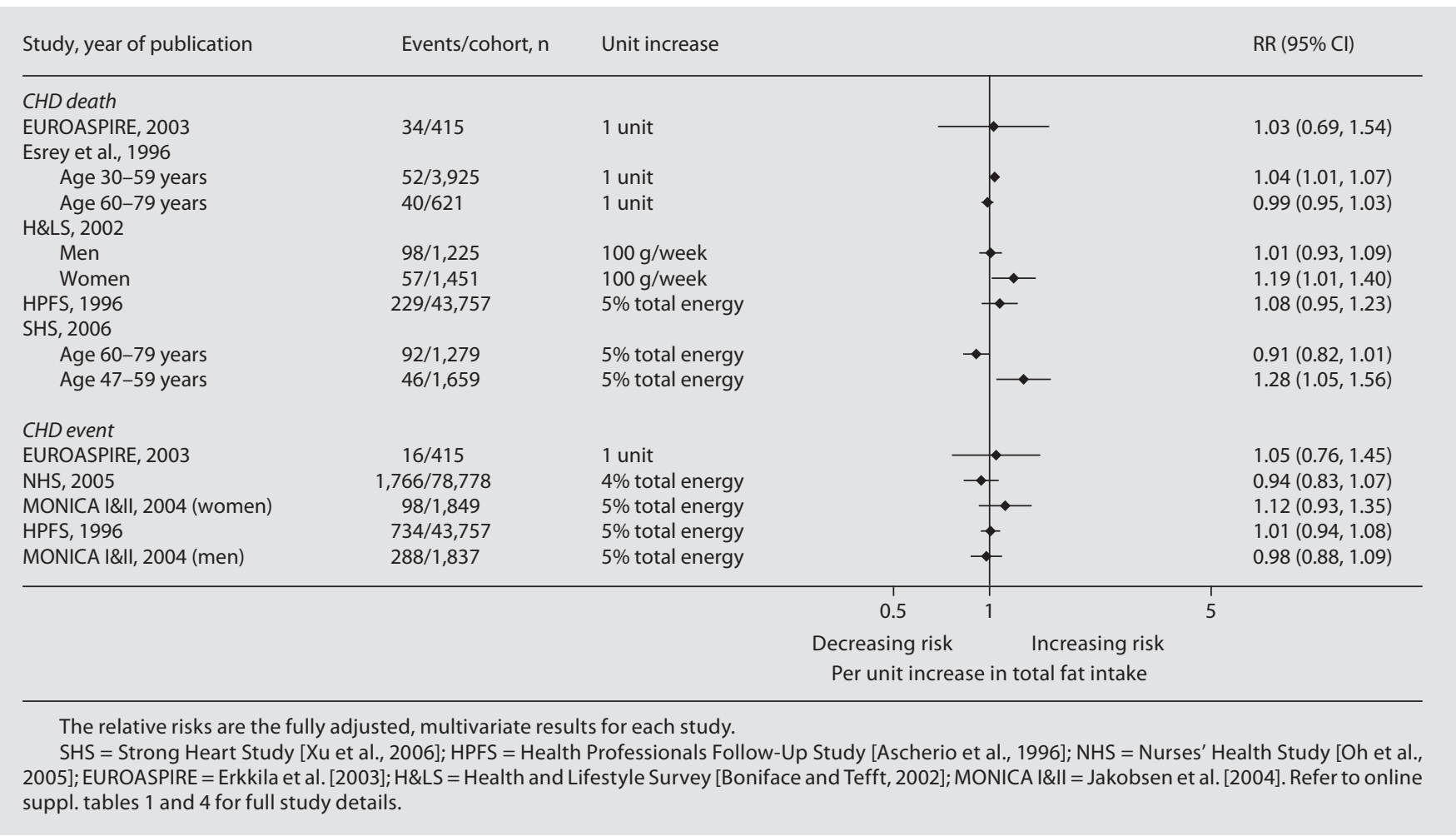

Fig. 2. RRs for CHD per unit increase in total fat intake. 


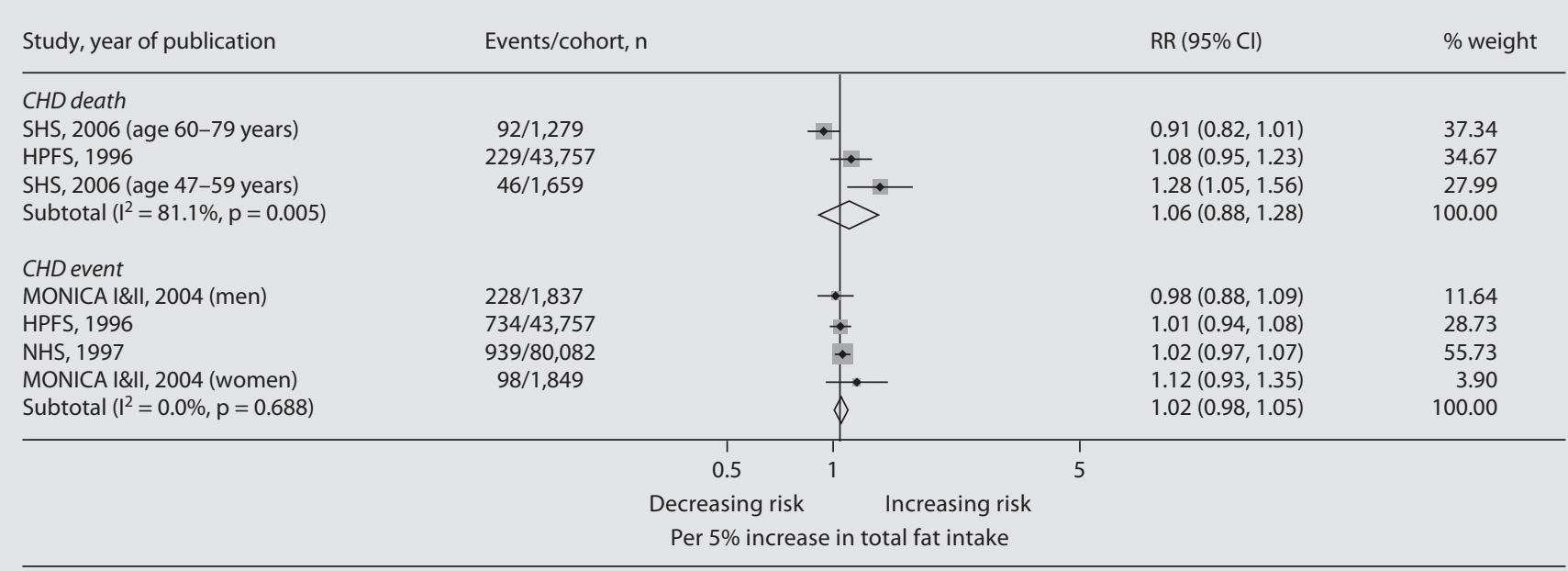

$p=0.517$ for CHD death subtotal; $p=0.404$ for CHD event subtotal. The relative risks are the fully adjusted, multivariate results for each study.

SHS = Strong Heart Study [Xu et al., 2006]; HPFS = Health Professionals Follow-Up Study [Ascherio et al., 1996]; NHS = Nurses' Health Study [Hu et al., 1997]; MONICA I\&II = Jakobsen et al. [2004]. Refer to online suppl. tables 1 and 4 for full study details.

Fig. 3. Meta-analysis of CHD risk per $5 \%$ increase in total fat intake.

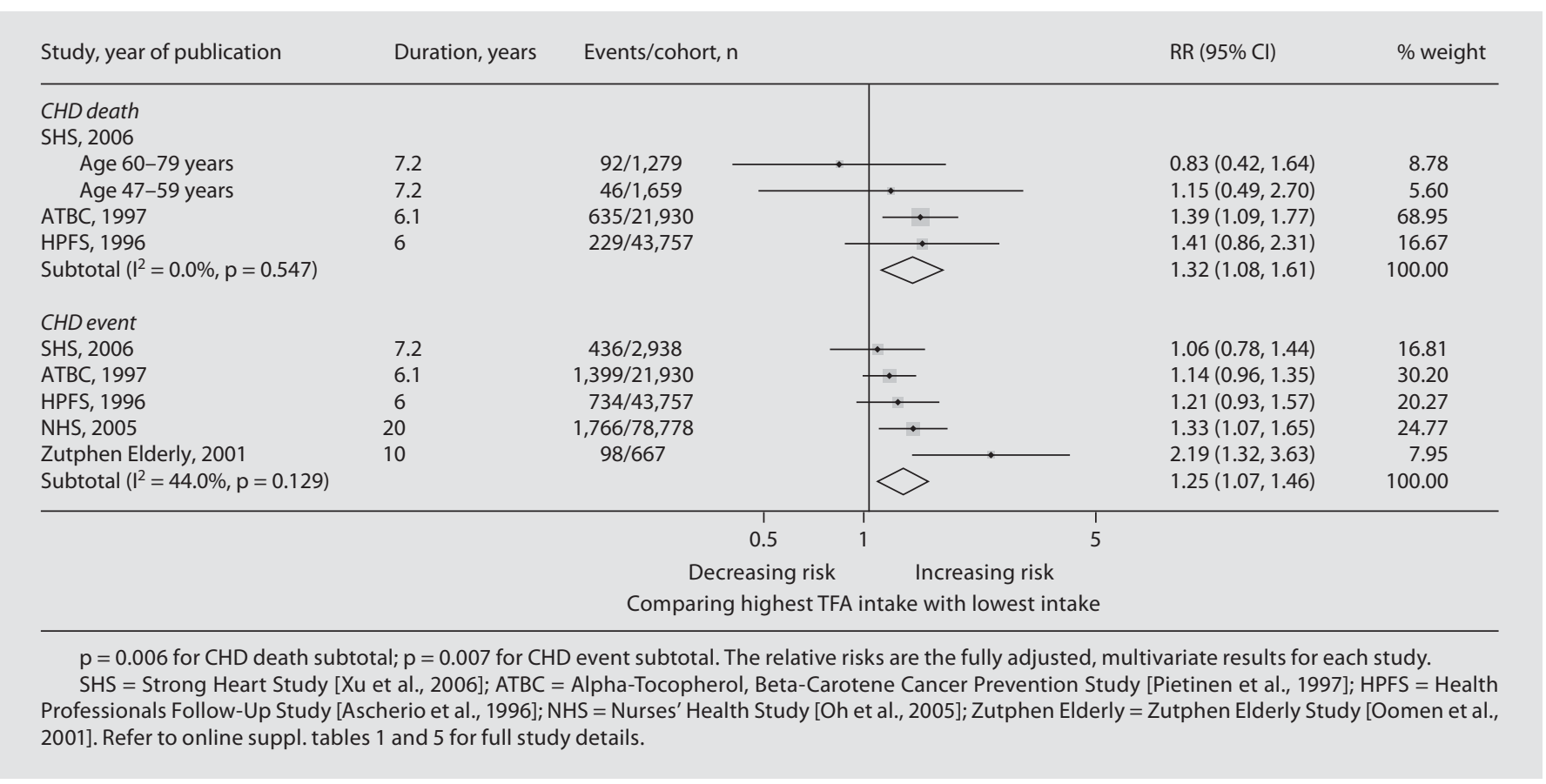

Fig. 4. Meta-analysis of prospective cohorts for TFA and CHD. 
Meta-Analysis of Cohort Studies of TFA and CHD

Intake of TFA was strongly associated with CHD mortality, with a RR of CHD death of 1.32 (95\% CI 1.08-1.61, $\mathrm{p}=0.006)$ for the highest compared with the lowest category (fig. 4). Similarly, high compared with low TFA intake was associated with a significantly increased risk of CHD events (RR 1.25, 95\% CI 1.07-1.46, $\mathrm{p}=0.007$ ). A $2 \%$ increase in TFA intake was associated with significantly higher risk of CHD events (RR 1.22, 95\% CI 1.11-1.35, $\mathrm{p}<0.001$ ) but not with CHD mortality (RR 1.21, 95\% CI $0.89-1.65, \mathrm{p}=0.227$; fig. 5). For the cohort studies included in the meta-analysis, mean or median TFA intake varied from 0.8 to $2.4 \%$ TE in the lowest category to 1.6 to $6.4 \% \mathrm{TE}$ in the highest category (table 3). Overall, the mean or median TFA intake varied from 2.0 to $4.3 \%$ TE in all cohorts (online suppl. table 5).

Meta-Analysis of Cohort Studies of SFA and CHD

Intake of SFA was not significantly associated with CHD mortality, with a RR of 1.14 (95\% CI 0.82-1.60, $\mathrm{p}=$ $0.431)$ for those in the highest compared with the lowest category of SFA intake (fig. 6). Similarly SFA intake was not significantly associated CHD events (RR 0.93, 95\% CI $0.83-1.05, p=0.269$ for high vs. low categories). Moreover, there was no significant association with $\mathrm{CHD}$ death (RR 1.11, 95\% CI 0.75-1.65, $\mathrm{p}=0.593$ ) per 5\% TE increment in SFA intake (fig. 8). For the cohort studies included in the meta-analysis, mean or median SFA intake varied from 7 to $11 \%$ TE in the lowest category to 14 to $18 \% \mathrm{TE}$ in the highest category (table 3 ). Overall the mean or median SFA intake in all cohort studies varied from 9 to $20 \%$ TE (online suppl. table 7 ).

Meta-Analysis of Cohort Studies of MUFA and CHD Intake of MUFA was not significantly associated with CHD mortality, with a RR of 0.85 (95\% CI 0.60-1.20, p = 0.356 ) for those in the highest compared with the lowest category of MUFA intake (fig. 9). Similarly, MUFA intake was not associated with CHD events (RR 0.87, 95\% CI $0.74-1.03, \mathrm{p}=0.110$, for high compared with low categories). Furthermore, there were no significant associations with CHD death (RR 0.92, 95\% CI 0.64-1.34, $\mathrm{p}=0.670$ ) or CHD events (RR 0.93, 95\% CI 0.77-1.12, $\mathrm{p}=0.449$ ) per 5\% TE increment in MUFA intake (fig. 11). For the cohort studies included in the meta-analysis, mean or median MUFA intake varied from 9 to $11 \%$ TE in the lowest category to 16 to $20 \% \mathrm{TE}$ in the highest category (table 3). Overall, the mean or median MUFA intakes in all cohort studies varied from 13 to $20 \%$ TE (online suppl. table 10).

Dietary Fat and Coronary Heart Disease
Meta-Analysis of Cohort Studies of PUFA and CHD

Intake of PUFA was strongly significantly associated with CHD mortality, with a RR of 1.25 (95\% CI 1.06-1.47, $\mathrm{p}=0.009$ ) for the highest compared with the lowest category (fig. 12). Conversely, high compared with low PUFA intake was not associated with CHD events (RR 0.97, 95\% CI $0.74-1.27, \mathrm{p}=0.825$, for high compared with low category). A 5\% incremental increase in PUFA intake was associated with a significantly lower risk of CHD events (RR 0.84, 95\% CI 0.70-1.00, p = 0.049), but not with CHD mortality ( $p=0.669$; fig. 14 ). For the cohort studies included in the meta-analysis, mean or median PUFA varied from 3 to $4 \% \mathrm{TE}$ in the lowest category to 6 to $10 \% \mathrm{TE}$ in the highest category (table 3). Overall, the mean or median PUFA intake in all cohort studies varied from 3 to $7 \%$ TE (online suppl. table 13).

The association between linoleic acid intake and risk of CHD was reported in the ATBC cohort [Pietinen et al., 1997], the Health Professionals Follow-up Study [Ascherio et al., 1996] and the EUROASPIRE study [Erkkila et al., 2003]. The results mirrored those of total PUFA; intake of linoleic acid was significantly associated with CHD mortality for those in the highest category compared with the lowest category of linoleic intake (1.25, 95\% CI 1.02-1.52, $\mathrm{p}=0.032$ ). Alternatively, linoleic acid intake was not associated with CHD events (RR 1.05, 95\% CI 0.92-1.20, p = 0.474 , for highest vs. lowest category; fig. 15).

Intake of $\alpha$-linolenic acid was not associated with $\mathrm{CHD}$ death (RR 0.84, 95\% CI 0.53-1.31, p = 0.439) or CHD events (RR 1.05, 95\% CI 0.78-1.42, p = 0.730) for those in the highest compared with the lowest category of intake (fig. 16). Mean $\alpha$-linolenic acid intake varied from 0.7 to $0.9 \mathrm{~g} /$ day in the lowest category to 1.4 to $2.5 \mathrm{~g} /$ day in the highest category (online suppl. table 13). In the Zutphen cohort, $\alpha$-linolenic acid intake in the lowest category was $0.4 \% \mathrm{TE}$ and in the highest category $0.67 \% \mathrm{TE}$.

\section{Meta-Analysis of Cohort Studies of n-3 LCPUFA} and CHD

For cohort studies included in the meta-analysis of n-3 LCPUFA and CHD there were about 5,361 CHD deaths amongst the 256,000 participants during approximately 4 million person-years of follow-up. CHD mortality rates ranged from approximately 12 to 1,100 deaths per 100,000 person years. The longest period of follow-up was 40 years and the shortest was 5 years. The studies were conducted in North American and European countries with the exception of 3 studies in Japan. Men accounted for more than $80 \%$ of the person-years of follow-up. The age at recruitment varied from 40 to 65 years (online suppl. table 16).

Ann Nutr Metab 2009;55:173-201 


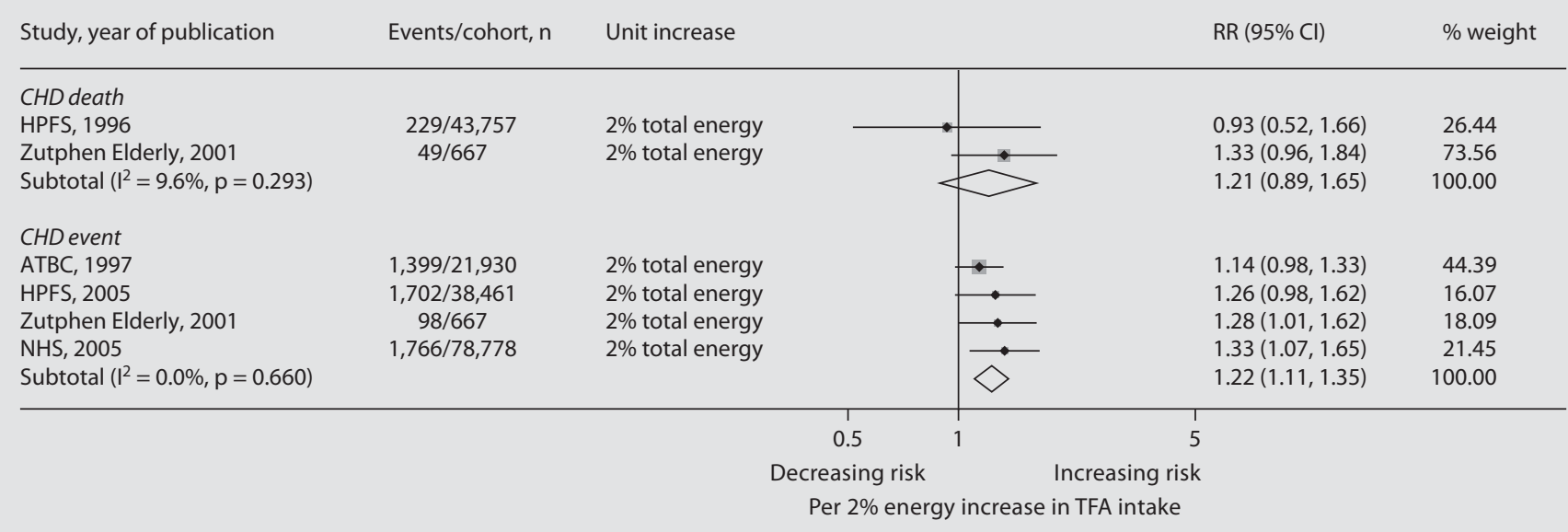

$p=0.227$ for $\mathrm{CHD}$ death subtotal; $p<0.001$ for CHD event subtotal. The relative risks are the fully adjusted, multivariate results for each study.

ATBC = Alpha-Tocopherol, Beta-Carotene Cancer Prevention Study [Pietinen et al., 1997]; HPFS = Health Professionals Follow-Up Study [Ascherio et al., 1996; Mozaffarian et al., 2005]; NHS = Nurses' Health Study [Oh et al., 2005]; Zutphen Elderly = Zutphen Elderly Study [Oomen et al., 2001]. Refer to online suppl. tables 1 and 6 for full study details.

Fig. 5. $\mathrm{RR}$ of $\mathrm{CHD}$ for a $2 \%$ energy increase in TFA.

Study, year of publication

Duration, years Events/cohort, $\mathrm{n}$

$\operatorname{RR}(95 \% \mathrm{Cl})$

$\%$ weight

CHD death

EUROASPIRE, 2003

Framingham, 1991 (age 45-55 years)

SHS, 2006 (age 60-79 years)

ATBC, 1997

Framingham, 1991 (age 56-65 years)

HPFS, 1996

Mann et al., 1997

SHS, 2006 (age 47-59 years)

Subtotal $\left(I^{2}=72.1 \%, p=0.001\right)$

$\begin{array}{cc} & \\ 5 & 34 / 415 \\ 16 & 99 / 420 \\ 7.2 & 92 / 1,279 \\ 6.1 & 635 / 21,930 \\ 16 & 114 / 393 \\ 6 & 229 / 43,757 \\ 13.3 & 64 / 10,802 \\ 7.2 & 46 / 1,659\end{array}$

$\longrightarrow$

CHD event

EUROASPIRE, 2003

ATBC, 1997

HPFS, 1996

NHS, 2005

SHS, 2006

Subtotal $\left(I^{2}=0.09 \%, p=0.673\right)$

$46 / 1,659$

$0.34(0.09,1.28)$

4.93

$0.78(0.61,1.00)$

19.11

(0.80 $\quad 11.57$

$0.93(0.60,1.44) \quad 15.64$

$0.99(0.77,1.27) \quad 19.02$

$1.72(1.01,2.93) \quad 13.89$

$2.77(1.25,6.14) \quad 9.72$

$5.17(1.64,16.30) \quad 6.13$

$1.14(0.82,1.60) \quad 100.00$

$34 / 415$

$1,399 / 21,930$

$734 / 43,757$

$1,766 / 78,778$

$436 / 2,938$
$0.71(0.29,1.74) \quad 1.77$

$0.87(0.73,1.04) \quad 46.18$

$0.96(0.73,1.26) \quad 18.95$

$0.97(0.73,1.29) \quad 17.59$

$1.11(0.82,1.50) \quad 15.50$

$0.93(0.83,1.05) \quad 100.00$

Decreasing risk Increasing risk

Comparing highest SFA intake to lowest intake

$p=0.431$ for CHD death subtotal; $p=0.269$ for CHD event subtotal. The relative risks correspond to comparisons of the highest SFA fat intakes with the lowest intakes, except the Framingham Study, which compared the sample mean SFA intakes with the National Cholesterol Education Program Recommendations. The EUROASPIRE relative risks correspond to comparisons of the highest cholesterol ester SFA fatty acid with the lowest cholesterol ester SFA fatty acid concentrations (mol\%). The relative risks are the fully adjusted, multivariate results for each study.

Framingham = Framingham Study [Posner et al., 1991]; SHS = Strong Heart Study [Xu et al., 2006]; ATBC = Alpha-Tocopherol, Beta-Carotene Cancer Prevention Study [Pietinen et al., 1997]; HPFS = Health Professionals Follow-Up Study [Ascherio et al., 1996]; NHS = Nurses' Health Study [Oh et al., 2005]; EUROASPIRE, $2003=$ Erkkila et al. [2003]. Refer to online suppl. tables 1 and 8 for full study details.

Fig. 6. Meta-analysis of prospective cohorts for saturated fat intake and CHD. 


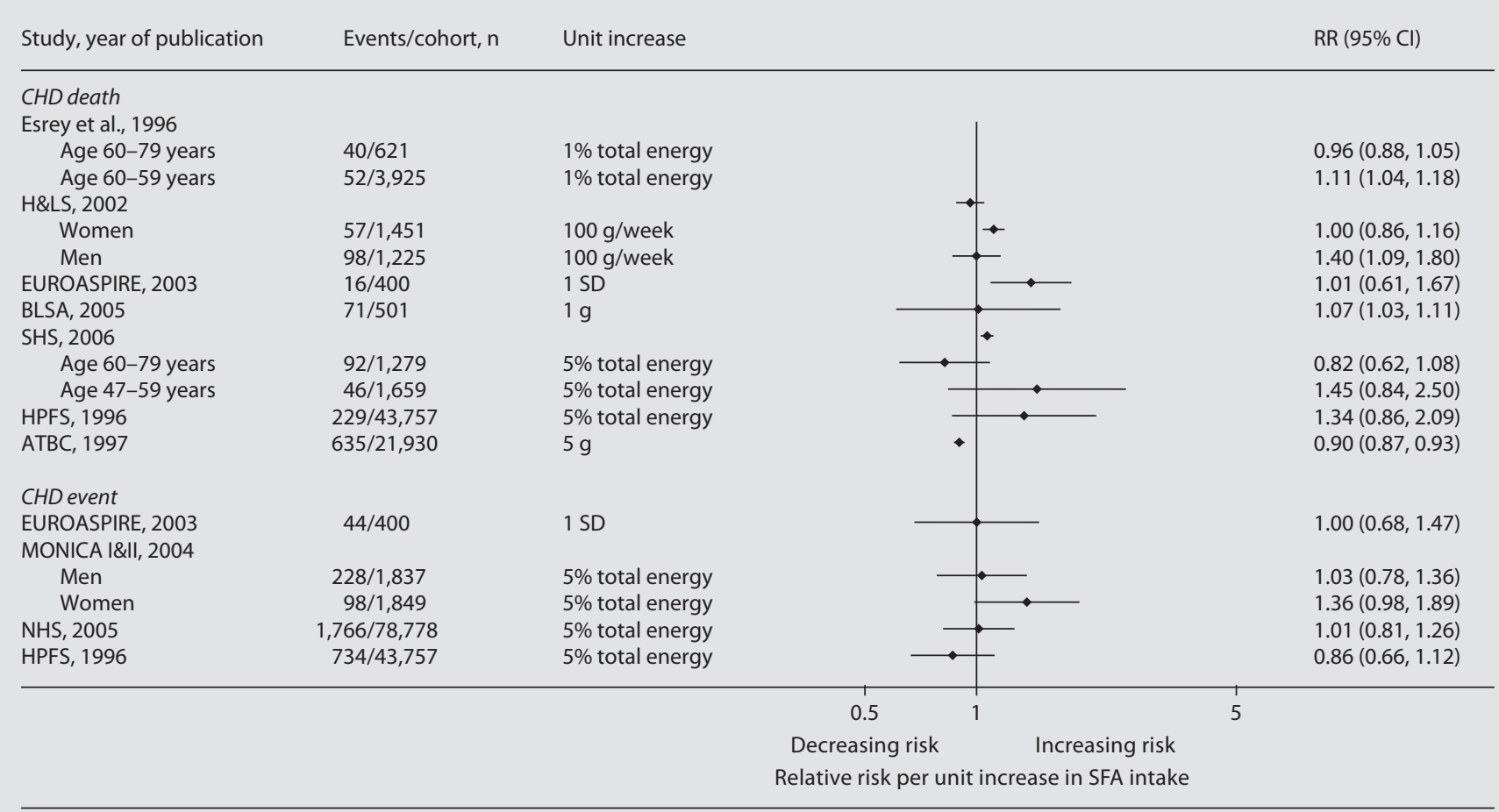

The relative risks are the fully adjusted, multivariate results for each study.

ATBC = Alpha-Tocopherol, Beta-Carotene Cancer Prevention Study [Pietinen et al., 1997]; BLSA = Baltimore Longitudinal Study of Aging [Tucker et al., 2005]; H\&LS = Health and Lifestyle Survey [Boniface and Tefft, 2002]; HPFS = Health Professionals Follow-Up Study [Ascherio et al., 1996]; SHS = Strong Heart Study [Xu et al., 2006]; MONICA I\&II = Jakobsen et al. [2004]; NHS = Nurses' Health Study [Oh et al., 2005]; EUROASPIRE, $2003=$ Erkkila et al. [2003]. Refer to online suppl. tables 1 and 9 for full study details.

Fig. 7. $\mathrm{RR}$ of $\mathrm{CHD}$ per unit increase in saturated fat intake.

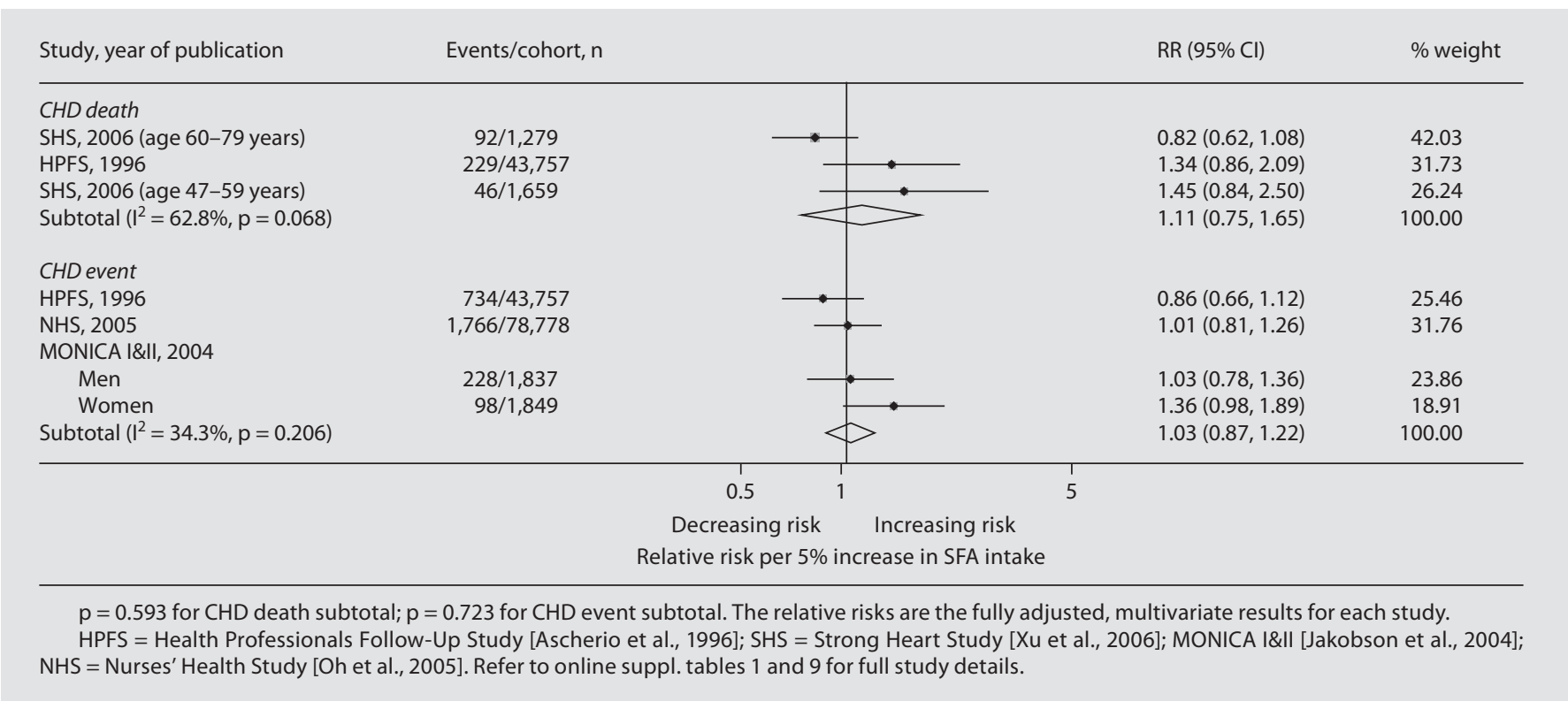

Fig. 8. Meta-analysis of CHD risk per each $5 \%$ of energy increase in saturated fat intake. 


\begin{tabular}{|c|c|c|c|c|c|c|}
\hline Study, year of publication & Duration, years & Events/coho & & & $\mathrm{RR}(95 \% \mathrm{Cl})$ & $\%$ weight \\
\hline \multicolumn{7}{|l|}{ CHD death } \\
\hline SHS, 2006 (age 60-79 years) & 7.2 & $92 / 1,279$ & - & & $0.54(0.27,1.08)$ & 14.09 \\
\hline Framingham, 1991 (age 45-55 years) & 16 & $99 / 420$ & $\longrightarrow$ & & $0.64(0.48,0.85)$ & 26.45 \\
\hline ATBC, 1997 & 6.1 & $635 / 21,930$ & $\longrightarrow$ & & $0.79(0.56,1.11)$ & 24.52 \\
\hline Framingham, 1991 (age 56-65 years) & 16 & $114 / 393$ & & - & $1.02(0.78,1.33)$ & 27.09 \\
\hline SHS, 2006 (age $47-59$ years) & 7.2 & $46 / 1,659$ & & $\longrightarrow$ & $3.42(1.17,10.03)$ & 7.85 \\
\hline Subtotal $\left(I^{2}=70.9 \%, p=0.008\right)$ & & & & & $0.85(0.60,1.20)$ & 100.00 \\
\hline \multicolumn{7}{|l|}{ CHD event } \\
\hline ATBC, 1997 & 6.1 & $1,399 / 21,930$ & $\rightarrow$ & & $0.82(0.69,0.97)$ & 50.73 \\
\hline NHS, 2005 & 20 & $1,766 / 78,778$ & $\longrightarrow$ & & $0.82(0.62,1.08)$ & 26.59 \\
\hline SHS, 2006 & 7.2 & $436 / 2,938$ & 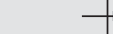 &. & $1.09(0.80,1.49)$ & 22.68 \\
\hline \multirow{4}{*}{ Subtotal $\left(I^{2}=27.4 \%, p=0.252\right)$} & & & $\diamond$ & & $0.87(0.74,1.03)$ & 100.00 \\
\hline & & & $0.5 \quad 1$ & $1 \quad 5$ & & \\
\hline & \multicolumn{6}{|c|}{ Decreasing risk Increasing risk } \\
\hline & \multicolumn{6}{|c|}{ Comparing highest MUFA intake with lowest intake } \\
\hline
\end{tabular}

$p=0.356$ for CHD death subtotal; $p=0.110$ for CHD event subtotal. The relative risks correspond to comparisons of the highest total fat intakes with the lowest intakes, except the Framingham Study, which compared the sample mean fat intakes with the National Cholesterol Education Program Recommendations. The relative risks are the fully adjusted, multivariate results for each study.

ATBC = Alpha-Tocopherol, Beta-Carotene Cancer Prevention Study [Pietinen et al., 1997]; HPFS = Health Professionals Follow-Up Study [Ascherio et al., 1996]; SHS = Strong Heart Study [Xu et al., 2006]; NHS = Nurses' Health Study [Oh et al., 2005]; Framingham = Framingham study [Posner et al., 1991]. Refer to online suppl. tables 1 and 11 for full study details.

Fig. 9. Meta-analysis of prospective cohorts for MUFA intake and CHD.

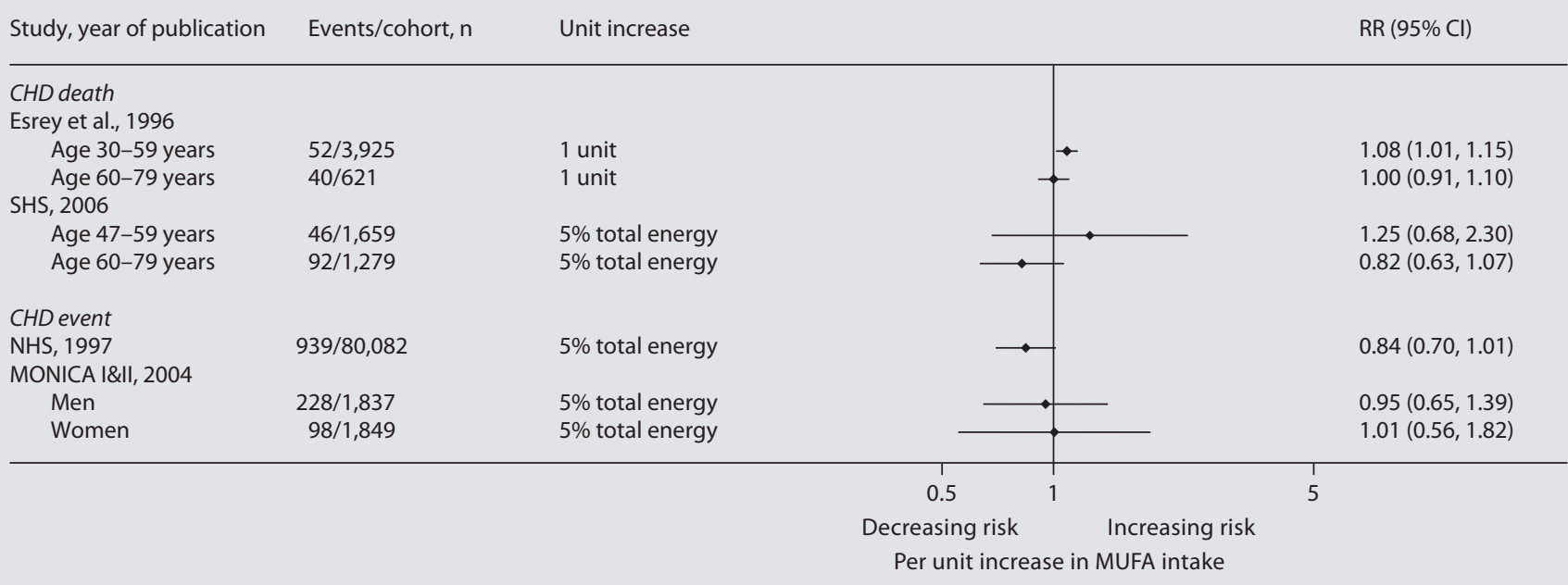

The relative risks are the fully adjusted, multivariate results for each study.

SHS = Strong Heart Study [Xu et al., 2006]; MONICA I\&II = Jakobsen et al. [2004]; NHS = Nurses' Health Study [Hu et al., 1997]. Refer to online suppl. tables 1 and 12 for full study details.

Fig. 10. RR of CHD per unit increase in MUFA intake. 


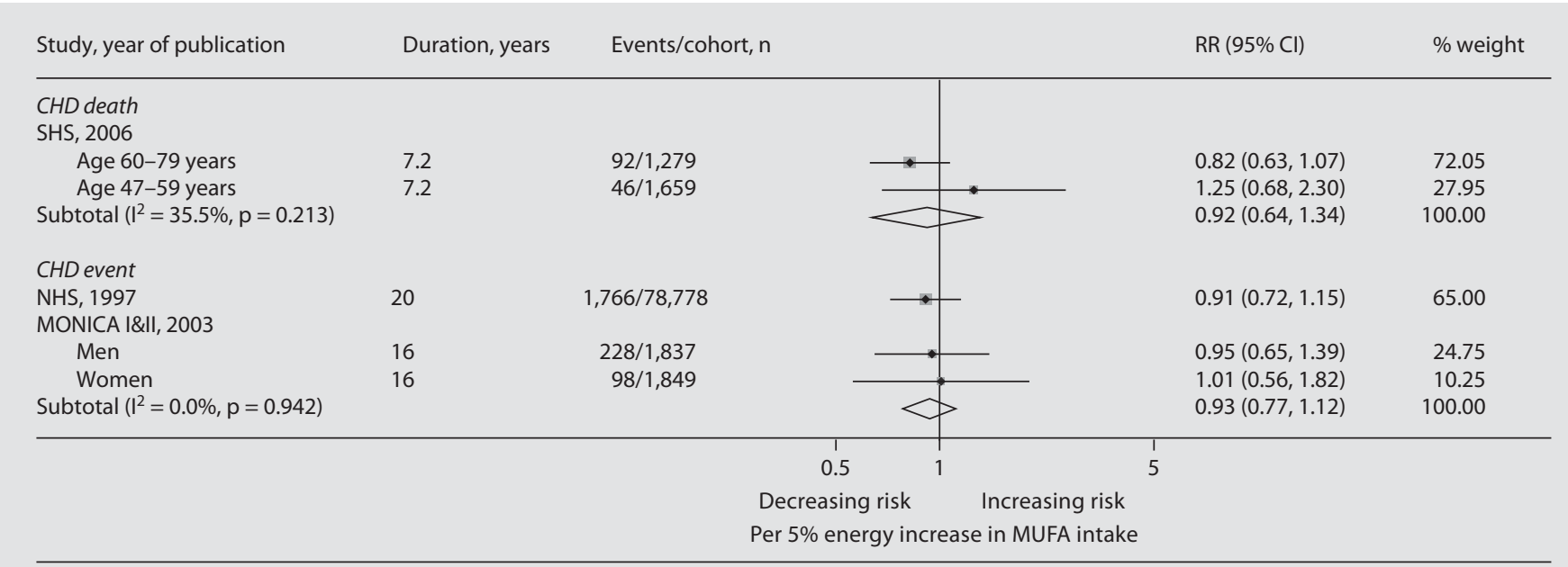

$p=0.670$ for CHD death subtotal; $p=0.449$ for CHD event subtotal. The relative risks are the fully adjusted, multivariate results for each study.

SHS = Strong Heart Study [Xu et al., 2006]; MONICA I\&II = Jakobsen et al., [2004]; NHS = Nurses' Health Study [Hu et al., 1997]. Refer to online suppl. tables 1 and 12 for full study details.

Fig. 11. RR of CHD per 5\% energy intake of MUFA intake.

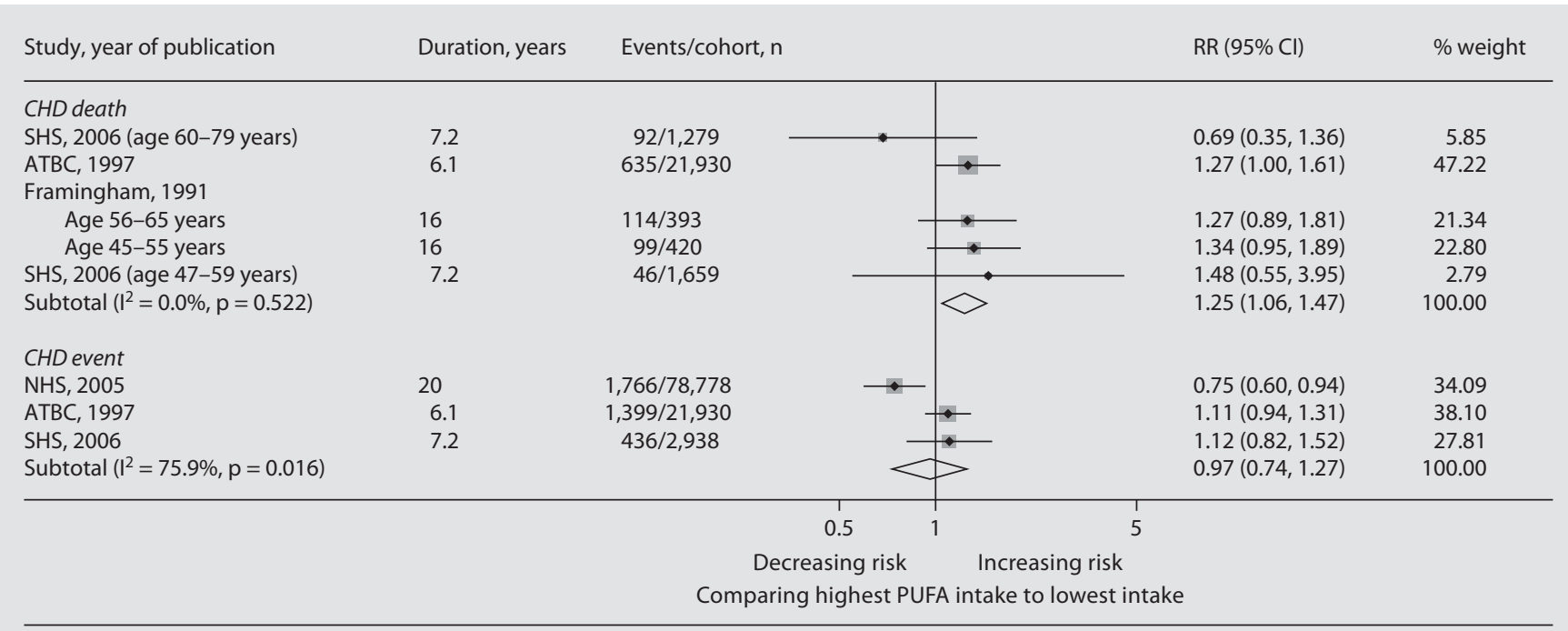

$p=0.009$ for CHD death subtotal; $p=0.825$ for CHD event subtotal. The relative risks correspond to comparisons of the highest PUFA intake with the lowest intakes, except the Framingham Study, which compared the sample mean fat intakes with the National Cholesterol Education Program Recommendations and risk of CHD. The relative risks are the fully adjusted, multivariate results for each study.

Framingham = Framingham Study [Posner et al., 1991]; SHS = Strong Heart Study [Xu et al., 2006]; ATBC = Alpha-Tocopherol, Beta-Carotene Cancer Prevention Study [Pietinen et al., 1997]; NHS = Nurses' Health Study [Oh et al., 2005]. Refer to online suppl. tables 1 and 14 for full study details.

Fig. 12. Meta-analysis of prospective cohorts for PUFA intake and CHD. 


\section{CHD death}

Esrey et al., 1996

Age $30-59$ years

Age 30-59 years

EUROASPIRE, 2003

SHS, 2006 (age 47-59 years)

HPFS, 1996

SHS, 2006 (age 60-79 years)

\section{$52 / 3,935$}

$40 / 621$

$16 / 415$

$46 / 1,659$

$229 / 43,757$

$92 / 1,279$

CHD event

EUROASPIRE, 2003

HPFS, 1996

MONICA I\&II, 2004 (men)

NHS, 1997

MONICA I\&Il, 2004 (women)

ATBC, 1997

$34 / 415$
$734 / 43,757$
$228 / 1,837$
$939 / 80,082$
$98 / 1,849$
$1,399 / 21,930$

1 unit

1 unit

$1 \mathrm{SD}$

$5 \%$ total energy

$5 \%$ total energy

$5 \%$ total energy

$1 \mathrm{SD}$

$5 \%$ total energy

$5 \%$ total energy

$5 \%$ total energy

$5 \%$ total energy

$5 \mathrm{~g}$
$0.99(0.90,1.09)$

$1.00(0.90,1.11)$

$0.92(0.55,1.54)$

$1.25(0.76,2.06)$

$0.93(0.52,1.66)$

$0.78(0.52,1.17)$

$1.08(0.78,1.50)$

$0.97(0.71,1.33)$

$0.80(0.55,1.16)$

$0.74(0.55,1.00)$

$0.89(0.50,1.58)$

$0.90(0.65,1.25)$

0.5

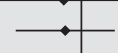

1

Decreasing risk Increasing risk

Relative risk per unit increase in PUFA intake

The relative risks are the fully adjusted, multivariate results for each study.

SHS = Strong Heart Study [Xu et al., 2006]; MONICA I\&II = Jakobsen et al. [2004]; NHS = Nurses' Health Study [Hu et al., 1997]; HPFS = Health Professionals Follow-Up Study [Ascherio et al., 1996]; EUROASPIRE, 2003 = Erkkila et al. [2003]. Refer to online suppl. tables 1 and 15 for full study details.

Fig. 13. RR of CHD per unit increase in PUFA intake.

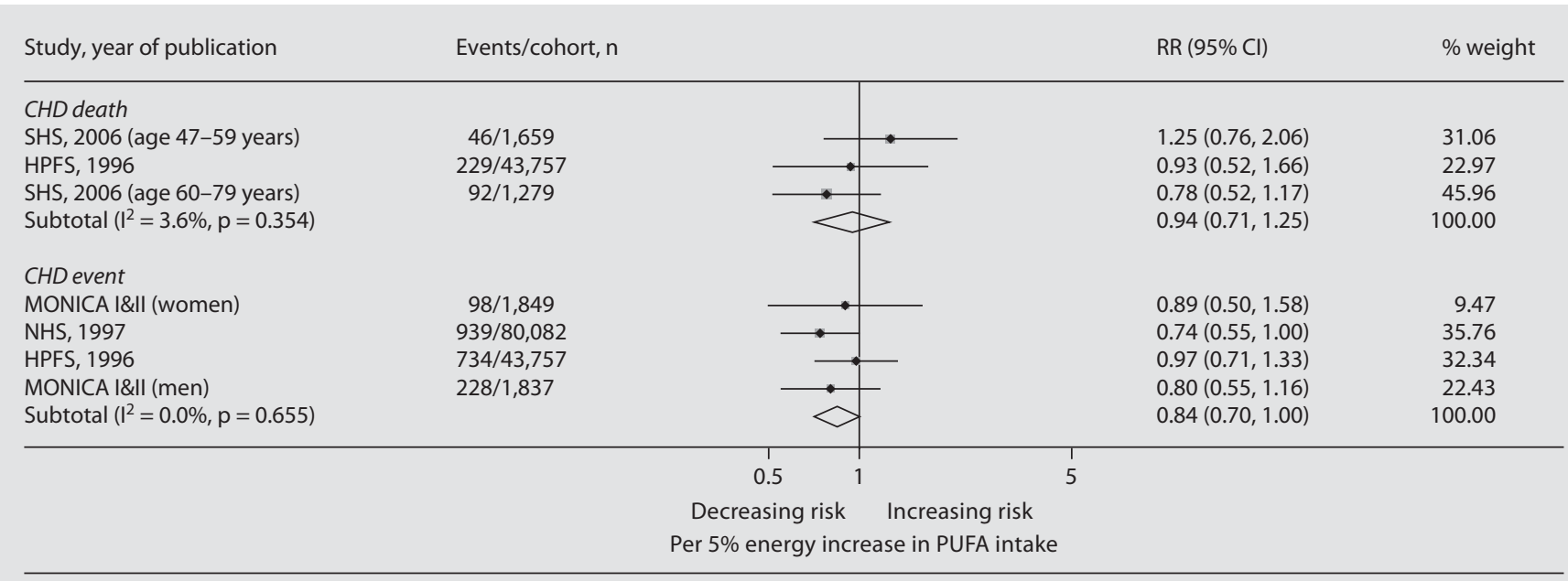

$p=0.669$ for $C H D$ death subtotal; $p=0.049$ for $C H D$ event subtotal. The relative risks are the fully adjusted, multivariate results for each study.

NHS = Nurses' Health Study [Hu et al., 1997]; MONICA I\&II = Jakobsen et al. [2004]; HPFS = Health Professionals Follow-Up Study [Ascherio et al., 1996]; SHS = Strong Heart Study [Xu et al., 2006]. Refer to online suppl. tables 1 and 15 for full study details.

Fig. 14. RR of CHD per 5\% energy increase in PUFA intake. 


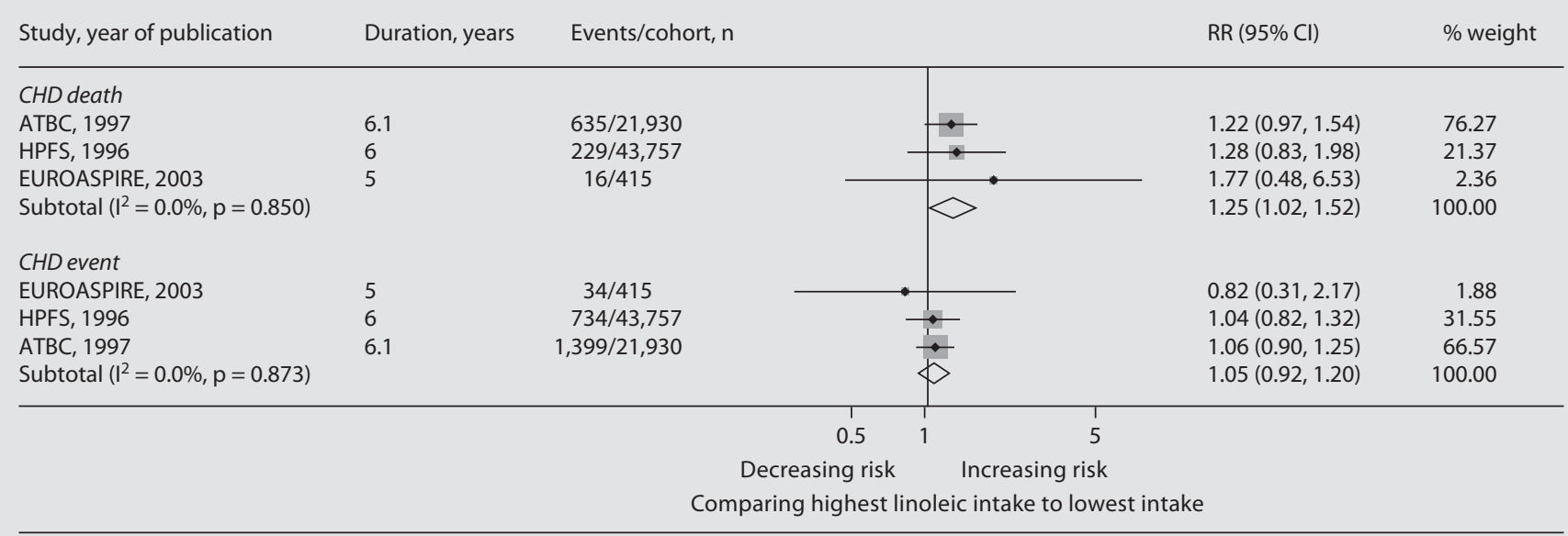

$p=0.032$ for CHD death subtotal; $p=0.474$ for CHD event subtotal. The relative risks correspond to comparisons of the highest linoleic fat intake with the lowest intake, except the EUROASPIRE Study, which compared the highest cholesterol ester linoleic concentrations with the lowest concentrations (mol\%).

ATBC = Alpha-Tocopherol, Beta-Carotene Cancer Prevention Study [Pietinen et al., 1997]; HPFS = Health Professionals Follow-Up Study [Ascherio et al., 1996]; EUROASPIRE = Erkkila et al. [2003]. Refer to online suppl. tables 1 and 14 for full study details.

Fig. 15. Meta-analysis of prospective cohorts for linoleic fatty acid intake and CHD.

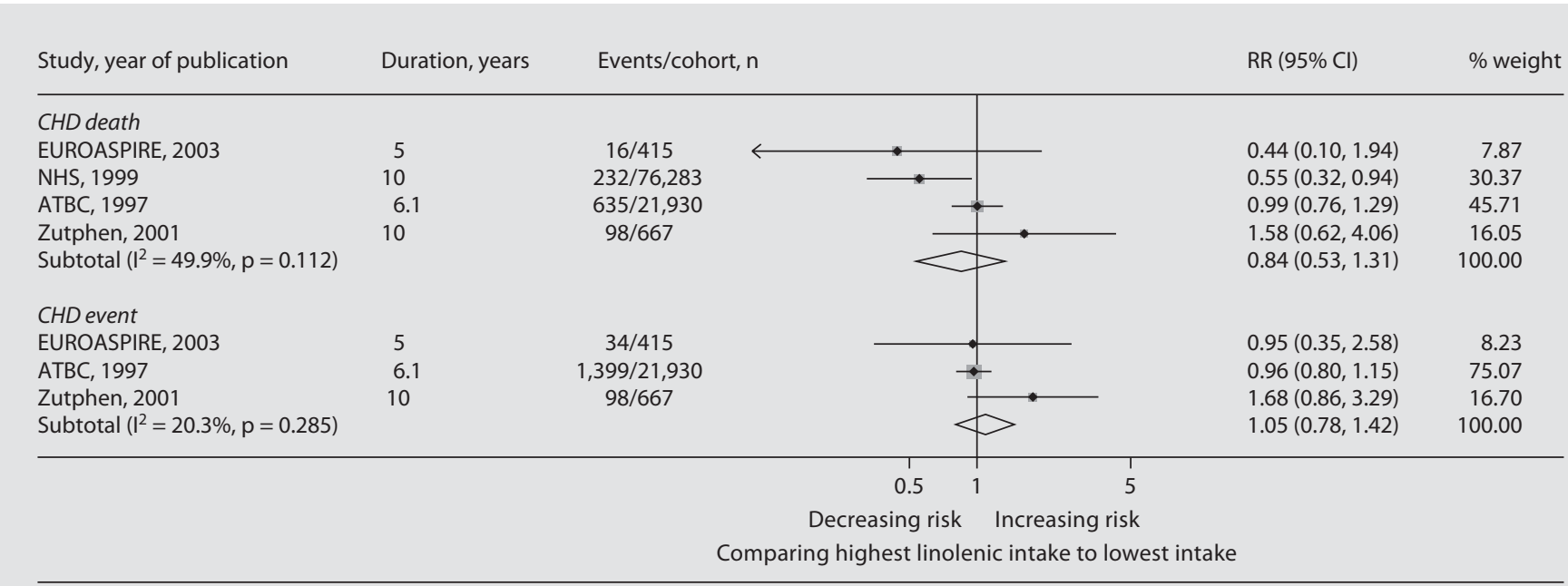

$p=0.439$ for CHD death subtotal; $p=0.730$ for CHD event subtotal. The relative risks correspond to comparisons of the highest $a$-linolenic fat intake with the lowest intake, except the EUROASPIRE Study, which compared the highest cholesterol ester a-linolenic concentrations with the lowest concentrations (mol\%).

NHS = Nurses' Health Study [Hu et al., 1999]; Zutphen = Zutphen Elderly Study [Oomen et al., 2001]; EUROASPIRE = Erkkila et al. [2003]; ATBC = AlphaTocopherol, Beta-Carotene Cancer Prevention Study [Pietinen et al., 1997]. Refer to online suppl. tables 1 and 14 for full study details.

Fig. 16. Meta-analysis of prospective cohorts for $\alpha$-linolenic fatty acid intake and CHD. 
Intake of $\mathrm{n}-3$ LCPUFA or fish consumption were strongly associated with CHD mortality (RR 0.82, 95\% CI $0.71-0.94, \mathrm{p}=0.006$ ) for the highest compared with the lowest category (fig. 17). Publication bias was discounted based on rejection of funnel plot asymmetry using the test developed by Begg and Mazumdar [1994]. Intake of n-3 LCPUFA was not associated with decreased risk of CHD events (RR 0.87, 95\% CI 0.71-1.06, $\mathrm{p}=0.157$ ), non-fatal CHD (RR 0.81, 95\% CI 0.59-1.10, p = 0.177) and total myocardial infarction (MI) (RR 0.79, 95\% CI 0.53$1.17, \mathrm{p}=0.235)$, for those in the highest category compared with the lowest category (fig. 18). Moreover, n-3 LCPUFA intake or fish consumption were not associated with sudden cardiac death (RR 0.62, 95\% CI 0.32-1.20, $\mathrm{p}=0.157$, for highest vs. lowest category; fig. 17). For the cohort studies included in the meta-analysis, mean or median n-3 LCPUFA intake varied from 0 to $0.3 \mathrm{~g}$ /day in the lowest category to 0.37 to $2.5 \mathrm{~g} /$ day in the highest category. The mean or median fish consumption varied from 0 to $23 \mathrm{~g} /$ day in the lowest category to 22 to $180 \mathrm{~g} /$ day in the highest category (table 3).

Randomised Controlled Trials of Dietary Fat and CHD Meta-Analysis of Randomised Controlled Trials of Fat-Modified Diets and CHD

The controlled trials included in the present metaanalysis of fat-modified diets and CHD risk were classified into 2 categories: (1) low-fat, and (2) altered P/S ratio. There were $331 \mathrm{CHD}$ deaths in the 2 low-fat trials. The duration of treatment varied from 3 to 8.1 years. There were $284 \mathrm{CHD}$ deaths in the $5 \mathrm{P} / \mathrm{S}$ trials. The duration of treatment varied from 2 to 5 years. The studies were conducted in North American and European countries. With the exception of the Womens' Health Initiative [Howard et al., 2006] the participants in the trials were men. The mean age of participants varied from 40 to 65 years. Selected characteristics of the individual trials are provided in online supplementary table 18 .

The results of the meta-analyses showed that the RR of fatal CHD was not reduced by either the low-fat diets $(1.00,95 \%$ CI $0.80-1.24, \mathrm{p}=0.317)$ or the high $\mathrm{P} / \mathrm{S}$ diets (0.84, 95\% CI $0.62-1.12, \mathrm{p}=0.867$ ), respectively (fig. 20; 21). There was no evidence of heterogeneity between the trials. High $\mathrm{P} / \mathrm{S}$ diets reduced the risk of total CHD events (RR 0.83, 95\% CI 0.69-1.00, p = 0.050), whereas the lowfat diets did not affect CHD events (RR 0.93, 95\% CI 0.84$1.04, \mathrm{p}=0.072$; fig. $20 ; 21$ ). There was evidence of heterogeneity between the low-fat trials but not between the P/S trials. Including results from the MRFIT trial - a trial in which the intervention was not restricted to a P/S diet - did not appreciably alter the pooled RR for CHD events, but the result was no longer statistically significant (RR $0.88,95 \%$ CI $0.77-1.01, \mathrm{p}=0.061$ ).

Restricting the meta-analysis to intervention trials of $\mathrm{P} / \mathrm{S}$ diets in which mean serum cholesterol concentration was significantly lower in the treatment group showed that the risk of fatal CHD was significantly reduced by the P/S diets (RR 0.52, 95\% CI 0.30-0.87, $\mathrm{p}=0.014$ ). Similarly, high $\mathrm{P} / \mathrm{S}$ diets reduced the risk of $\mathrm{CHD}$ events (RR $0.68,95 \%$ CI $0.49-0.94, \mathrm{p}=0.020$; fig. 22 ).

The low-fat diet did not alter the RR of all cause mortality during follow-up (RR 0.98, 95\% CI 0.90-1.06, p = 0.590 ), neither did the P/S diet (RR 0.88, 95\% CI $0.76-$ $1.02, \mathrm{p}=0.083$; fig. 19).

The Women's Health Initiative [Howard et al., 2006] involved 48,000 postmenopausal women aged 50-79 years, that were randomised to a low-fat (20\% TE) highfruit and vegetable diet or comparison group. The mean duration of follow-up was 8.1 years and total fat intake was $8.2 \%$ TE lower in the treatment than comparison group at 6 years. The P/S ratios of the diets in the treatment and comparison groups were not different. Serum and total cholesterol concentrations at 3 years were significantly but marginally lower in the low-fat diet, by 1.5 and $2.7 \%$, respectively. Weight was $1.29 \mathrm{~kg}$ lower in the diet group at 3 years. For all participants, the diet had no significant effect on CHD death (RR 1.02, 95\% CI 0.84-1.25) or nonfatal MI and CHD death (RR 0.98, 95\% CI 0.88-1.09). Similarly, for women with no history of cardiovascular disease the low-fat diet had no effect on CHD death (RR 1.01, 95\% CI 0.81-1.27) or nonfatal MI and CHD death (RR $0.93,95 \%$ CI $0.83-1.05)$. The results suggest that a low-fat diet in postmenopausal women does not reduce CHD risk, but lacked the power to refute this hypothesis.

The Lyon Diet and Heart Study [de Lorgeril et al., 1994] dietary intervention was a quasi Mediterranean diet which could not be classified as either a low-fat or altered $\mathrm{P} / \mathrm{S}$ intervention. The intervention led to large and significant reductions in the risk of $\mathrm{CHD}$ death and $\mathrm{CHD}$ events during the 2 years of follow-up, by 65 and $70 \%$, respectively; yet the magnitude of differences in diet composition, established risk factors for $\mathrm{CHD}$, or plasma fatty acids between the treatment and comparison groups were very small, in most cases they were not significant.

Meta-Analysis of Randomised Controlled Trials of n-3 LCPUFA or Fish and CHD

The meta-analysis included results from 16 randomised controlled trials (fig. 25; 26). We include the results from both DART trials [Burr et al., 1989, 2003] but excluded re- 


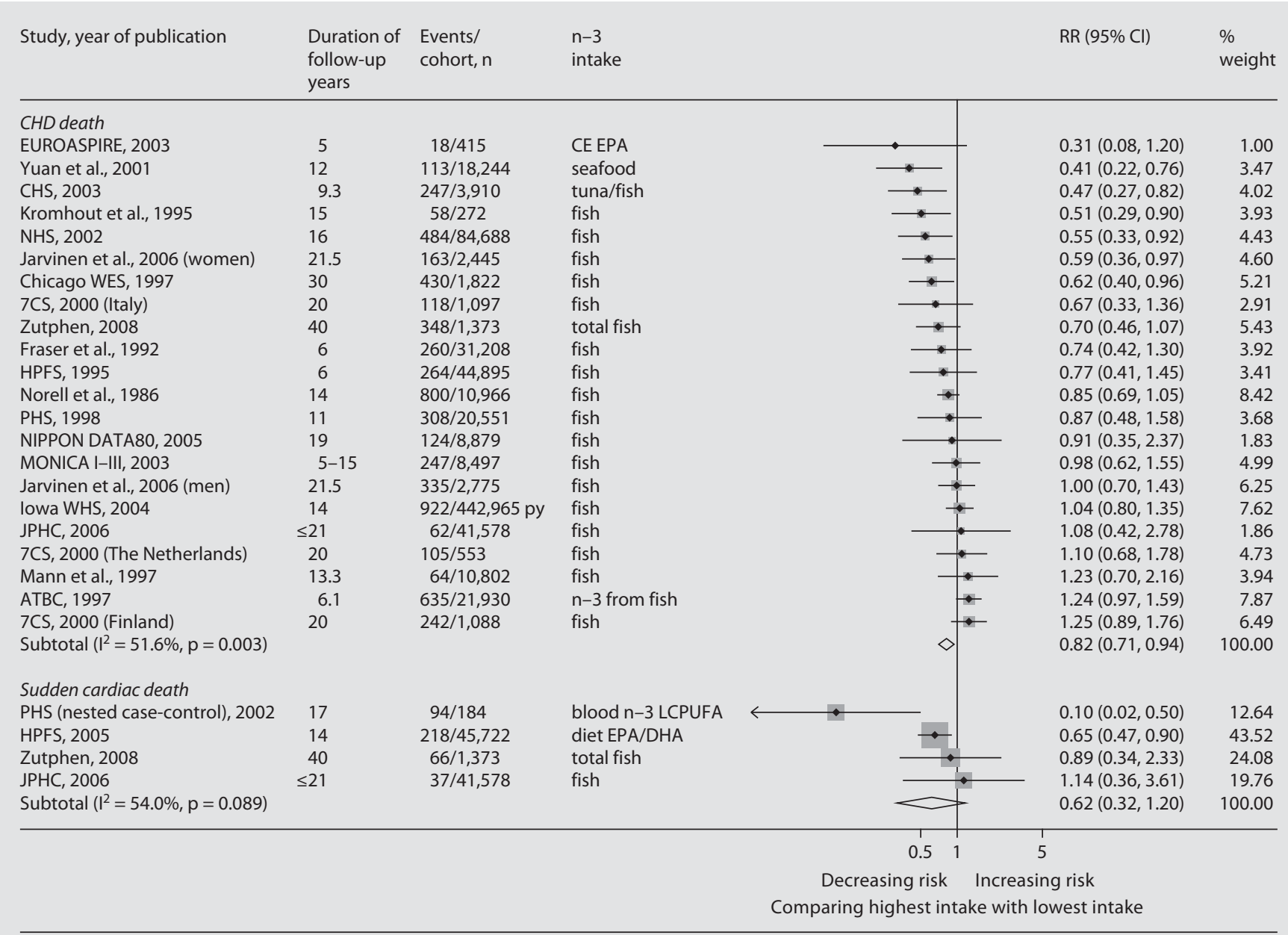

$p=0.006$ for CHD death subtotal; $p=0.157$ for sudden cardiac death

$\mathrm{CE}=$ Cholesterol ester; $\mathrm{DHA}=$ docosahexaenoic acid; $\mathrm{EPA}=$ eicosapentaenoic acid py = person-years.

EUROASPIRE = Erkkila et al. [2003]; CHS = Cardiovascular Health Study [Mozaffarian et al., 2003]; NHS = Nurses' Health Study [Hu et al., 2002]; Chicago WES = Chicago Western Electric Study [Daviglus et al., 1997]; 7CS = Seven Countries Study [Oomen et al., 2000]; HPFS = Health Professionals' Follow-Up Study [Ascherio et al., 1995]; PHS = Physicians' Health Study [Albert et al., 1998, 2002]; NIPPON DATA80 = Nakamura et al. [2005]; lowa WHS = lowa Women's Health Study [Folsom and Demissie, 2004]; JPHC = Japan Public Health Center-Based Study Cohort I [Iso et al., 2006]; ATBC = Alpha-Tocopherol Beta-Carotene Cancer Prevention Study [Pietinen et al., 1997]; Zutphen, 2008 = Zutphen Study, 2008 [Streppel et al., 2008]; MONICA I-III [Osler et al., 2003]. Refer to online suppl. tables 16 and 17 for full study details.

Fig. 17. Meta-analysis of prospective cohorts for fish or n-3 LCPUFA intake and fatal CHD.

sults from the trial by Singh et al. [Expression of concern, 2005]. There were about $1,300 \mathrm{CHD}$ deaths amongst 37,000 participants during 140,000 person-years of follow-up. Overall CHD mortality rates across the trials ranged from approximately 70 to over 4,000 per 100,000 person-years. Trial duration varied from 6 months to 9 years. The average duration of trials in which CHD death was monitored was 2 years. After excluding the JELIS trial [Yokoyama et al., 2007], men accounted for about $90 \%$ of the personyears of follow-up. In the JELIS trial, women made up 70\% of the participant population and only $60 \mathrm{CHD}$ deaths occurred during 5 years of follow-up amongst 18,645 patients. The most common form of treatment was fish oil supplements, though a few trials involved increased fish consumption [Burr et al., 1989, 2003]. Total intake of eicosapentaenoic acid (EPA) and docosahexaenoic acid (DHA) ranged from $0.5-6.9 \mathrm{~g} /$ day (online suppl. table 18).

The results of the meta-analysis showed that the RR of CHD death was not significantly reduced by $\mathrm{n}-3$ LCPUFA treatment, 0.88 (95\% CI 0.76-1.01, $\mathrm{p}=0.061$; 


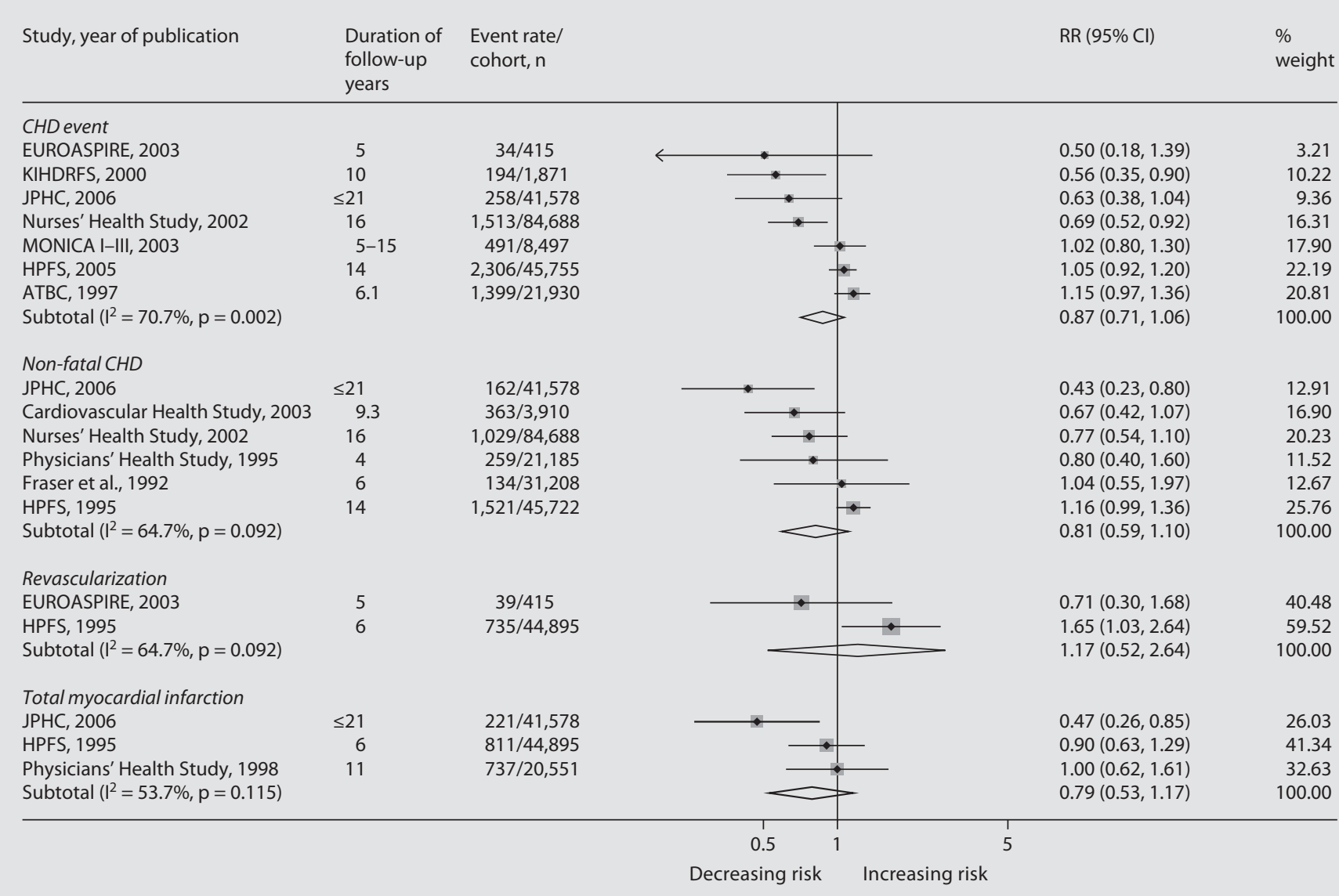

$p=0.157$ for CHD event; $p=0.177$ for non-fatal CHD subtotal; $p=0.700$ for revascularization; $p=0.235$ for total myocardial infarction subtotal. The relative risks correspond to comparisons of the highest intake of fish or marine $n-3$, or blood biomarker concentrations of EPA or EPA and DHA, with the lowest intakes or concentrations, as indicated on the forest plot. The relative risks are the fully adjusted, multivariate results for each study.

$\mathrm{CE}=$ Cholesterol ester; $\mathrm{DHA}=$ docosahexaenoic acid; $\mathrm{EPA}=$ eicosapentaenoic acid; $\mathrm{py}=$ person-years; $\mathrm{n} . \mathrm{n} .=$ not noted.

EUROASPIRE = Erkkila et al. [2003); CHS = Cardiovascular Health Study [Mozaffarian et al., 2003]; NHS = Nurses' Health Study [Hu et al., 2002]; Chicago WES = Chicago Western Electric Study [Daviglus et al., 1997]; HPFS = Health Professionals' Follow-Up Study [Ascherio et al., 1995; Mozaffarian et al., 2005]; PHS = Physicians' Health Study [Albert et al., 1998]; JPHC = Japan Public Health Center-Based Study Cohort I [lso et al., 2006]; ATBC = Alpha-Tocopherol Beta-Carotene Cancer Prevention Study [Pietinen et al., 1997]; KIHDRFS = Kuopio Ischemic Heart Disease Risk Factor Study [Rissanen et al., 2000]; MONICA I-III [Osler et al., 2003]. Refer to online suppl. tables 16 and 17 for full study details.

Fig. 18. Meta-analysis of prospective cohorts for fish or n-3 LCPUFA fat intake and non-fatal or total CHD.

fig. 25). Publication bias was discounted based on rejection of funnel plot asymmetry using the test developed by Begg and Mazumdar [1994]. The risks of fatal MI (RR $0.92,95 \%$ CI $0.65-1.29, \mathrm{p}=0.626)$ and sudden cardiac death (RR 1.02, 95\% CI 0.78-1.33, p = 0.889) were also not decreased by treatment. However, the RR of CHD events was significantly lowered with n-3 LCPUFA treatment $(0.89,95 \%$ CI $0.82-0.98, \mathrm{p}=0.012)$. Non-fatal CHD outcomes such as revascularization events (RR 0.94, 95\% CI
$0.86-1.04, \mathrm{p}=0.211)$, non-fatal MI (RR 1.03, 95\% CI 0.77$1.37, \mathrm{p}=0.864$ ), and angina (RR $0.89,95 \%$ CI $0.75-1.04$, $\mathrm{p}=0.149$ ) were not significantly reduced by $\mathrm{n}-3$ LCPUFA treatment (fig. 26).

Meta-analysis of study results after exclusion of the DART II trial considerably altered the summary estimates, such that n-3 LCPUFA significantly reduced the risk of fatal CHD (RR 0.81, 95\% CI 0.71-0.92, p = 0.001), fatal MI (RR 0.74, 95\% CI 0.57-0.96, p = 0.025), and CHD 


\begin{tabular}{|c|c|c|c|c|c|c|}
\hline Study, year of publication & $\begin{array}{l}\text { Duration } \\
\text { years }\end{array}$ & $\begin{array}{l}\text { Events/ } \\
\text { intervention, } \mathrm{n}\end{array}$ & $\begin{array}{l}\text { Events/ } \\
\text { control, } \mathrm{n}\end{array}$ & & $\mathrm{RR}(95 \% \mathrm{Cl})$ & $\%$ weigh \\
\hline \multicolumn{7}{|l|}{ SFA-PUFA intervention } \\
\hline STARS, 1992 & 3.25 & $1 / 27$ & $3 / 28$ & & $0.37(0.04,3.35)$ & 0.41 \\
\hline \multicolumn{7}{|l|}{ Finn Mental Hospital } \\
\hline Women & 3.7 & $415 / 3,598$ & $465 / 2,836$ & $\rightarrow$ & $0.73(0.65,0.83)$ & 19.69 \\
\hline Men & 4.3 & $187 / 2,276$ & $217 / 1,902$ & $\rightarrow$ & $0.74(0.62,0.89)$ & 16.58 \\
\hline Oslo Diet-Heart Study & 5 & $41 / 206$ & $55 / 206$ & $\rightarrow$ & $0.79(0.55,1.13)$ & 9.22 \\
\hline DART, 1989 & 2 & $111 / 1,018$ & $113 / 1,015$ & $\rightarrow$ & $0.98(0.77,1.26)$ & 13.61 \\
\hline Veterans' Administration Trial, 1969 & 3.7 & $174 / 424$ & $177 / 422$ & + & $0.98(0.83,1.17)$ & 17.12 \\
\hline MRC Soya Trial, 1968 & 4 & 27/199 & $25 / 194$ & ? & $1.05(0.63,1.75)$ & 5.84 \\
\hline Minnesota Coronary Survey, 1989 & 4.5 & $269 / 4,541$ & $248 / 4,516$ & ! & $1.07(0.91,1.27)$ & 17.53 \\
\hline Subtotal $\left(I^{2}=65.2 \%, p=0.005\right)$ & & & & $\diamond$ & $0.88(0.76,1.02)$ & 100.00 \\
\hline \multicolumn{7}{|l|}{ Low-fat diet intervention } \\
\hline London Low Fat, 1965 & 3 & 20/123 & $24 / 129$ & . & $0.89(0.52,1.54)$ & 2.08 \\
\hline WHI, 2006 & 8.1 & $950 / 19,541$ & $1,454 / 29,294$ & 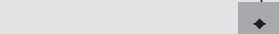 & $0.98(0.91,1.06)$ & 97.92 \\
\hline \multirow{3}{*}{ Subtotal $\left(1^{2}=0.0 \%, p=0.737\right)$} & & & & $\phi$ & $0.98(0.90,1.06)$ & 100.00 \\
\hline & & & & 0.5 & $\begin{array}{l}1 \\
5\end{array}$ & \\
\hline & & & & Favours intervention & Favours control & \\
\hline
\end{tabular}

$p=0.083$ for SFA-PUFA intervention subtotal; $p=0.590$ for low-fat diet intervention.

STARS = Watts et al. [1992]; Oslo Diet-Heart Study = Leren [1970]; DART = Burr et al. [1989]; MRC Soya Trial = Medical Research Council, [1968]; Minnesota Coronary Survey $=$ Frantz et al. [1989]; London Low Fat $=$ [Research Committee, 1965]; WHI = Howard et al. [2006]; Finn Mental Hospital (both women and men) = Miettinen et al. [1972]; Veterans' Administration Trial = Dayton and Pearce [1969]. Refer to online suppl. table 18 for full study details.

Fig. 19. Meta-analysis of fat modification trials and total mortality.

events (RR 0.89, 95\% CI 0.82-0.98, $\mathrm{p}=0.012$; fig. 27). The summary estimate of RR of sudden cardiac death was 0.89 (95\% CI $0.72-1.09, \mathrm{p}=0.251)$.

The RR of all cause mortality during the follow-up periods of the trials was not significantly lower in participants taking n-3 LCPUFA $(0.95,95 \%$ CI $0.87-1.03$, $\mathrm{p}=$ 0.225; fig. 23). Following exclusion of the DART II trial the RR of total mortality was 0.93 (95\% CI 0.86-0.99, p = 0.027; fig. 24). Publication bias was discounted based on rejection of funnel plot asymmetry using the test developed by Begg and Mazumdar [1994].

The results of recent meta-analysis [Jenkins et al., 2008] of 3 randomised controlled (1- to 2-year duration) trials of fish oil supplementation in patients with implantable cardioverter defibrillators showed no effect of $\mathrm{n}-3$ LCPUFA on the RR of defibrillator discharge (RR $0.93,95 \%$ CI $0.70-1.24, \mathrm{p}=0.63$ ).

\section{Discussion}

Differences between populations in the amount and type of fat consumed explain much of the variation in the incidence of cardiovascular diseases [Keys, 1980]. Ac- cording to the classic 'diet-heart' hypothesis, high intake of SFAs and cholesterol and low intake of PUFAs increase serum cholesterol levels and risk of CHD. However, few within-population studies have been able to demonstrate consistent associations with any specific dietary lipids, with the exception of trans fats and n-3 fatty acids. The available evidence from cohort and randomised controlled trials is unsatisfactory and unreliable to make judgement about and substantiate the effects of dietary fat on risk of CHD. The null results of the observational studies of dietary lipids and CHD do not negate the importance of the underlying associations, but reflect the combined effects of limitations of dietary assessment methods, inadequate numbers of participants studied and the prolonged follow-up of individuals. Furthermore, the evidence from cohort studies of dietary intake of fats and CHD is mostly unreliable (with a few exceptions) because most studies have ignored the effects of measurement error and regression dilution bias. Few studies attempted to measure the within-person variability or reproducibility of the categorizations of dietary fat when assessing these associations. Hence, the null results are very likely to result from regression dilution bias and confounding of 1 nutrient by another. By contrast, CHD risk is moderately 


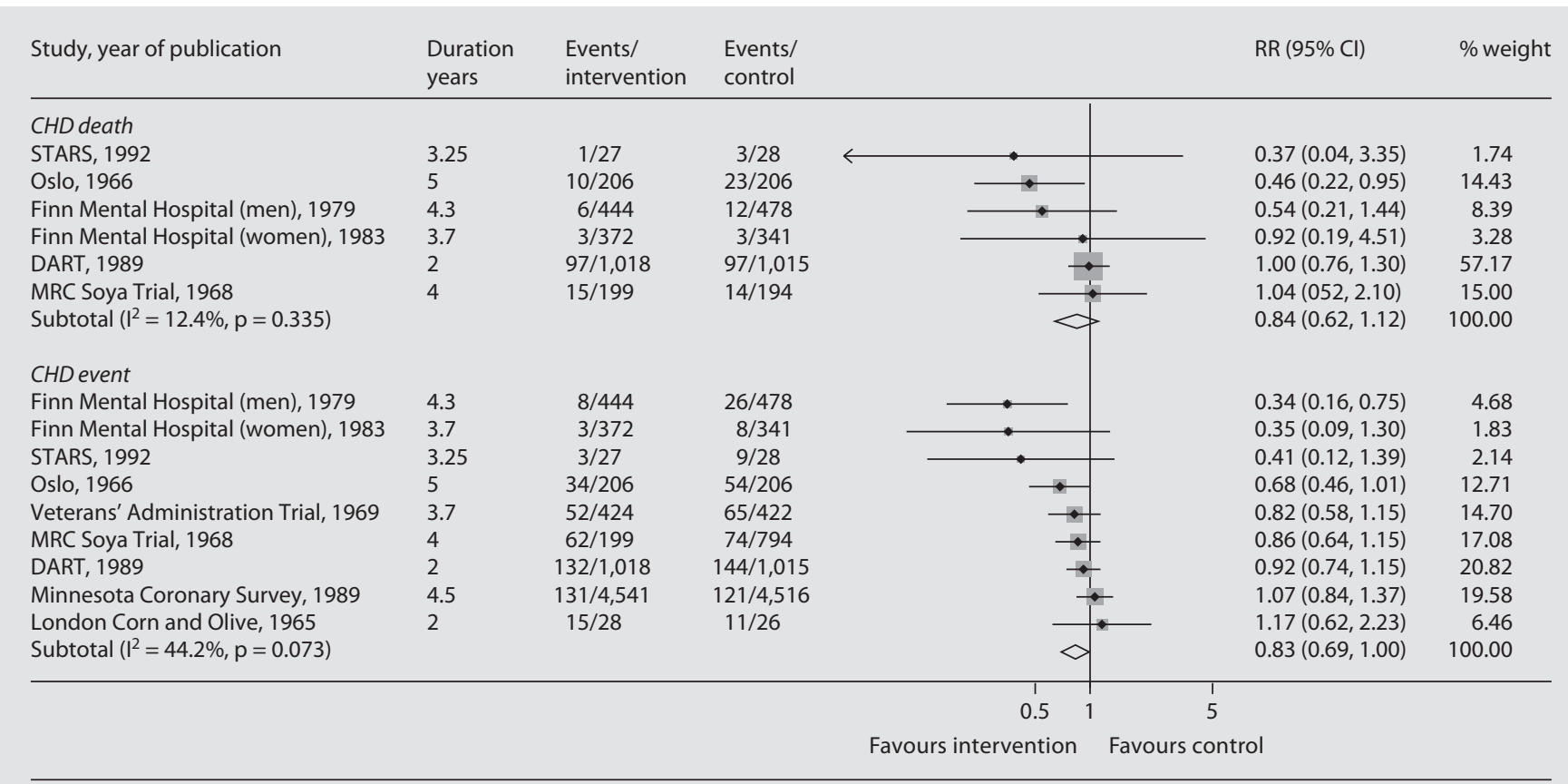

$p=0.867$ for CHD death subtotal; $p=0.050$ for CHD event subtotal.

STARS = Watts et al. [1992]; Oslo = Leren [1970], 5-year results were used; DART = Burr et al. [1989]; MRC Soya Trial = Medical Research Council [1968]; Minnesota Coronary Survey = Frantz et al. [1989]; Finnish Mental Hospital (men) $=$ Turpeinen [1979]; Finnish Mental Hospital (women) $=$ Miettinen et al [1983]; Veterans' Administration Trial = Dayton and Pearce [1969]; London Corn and Olive = Rose et al. [1965]. Refer to online suppl. table 18 for full study details.

Fig. 20. Meta-analysis of altered PUFA - SFA modified trials.

Table 4. Summary of the strength of evidence of dietary fat and $\mathrm{CHD}$

\begin{tabular}{lll}
\hline Type of fat & Fatal CHD & CHD events \\
\hline Total fat & C-NR & C-NR \\
TFA & P $\uparrow$ & C $\uparrow$ \\
SFA for CHO & P-NR & P-NR \\
$\begin{array}{l}\text { MUFA for SFA } \\
\text { PUFA for SFA }\end{array}$ & $\mathrm{C} \downarrow$ & $\mathrm{C} \downarrow$ \\
$\quad \begin{array}{l}\text { Linoleic } \\
\alpha \text {-linolenic }\end{array}$ & & \\
n-3 LCPUFA & $\mathrm{P} \downarrow$ & $\mathrm{C} \downarrow$ \\
\hline
\end{tabular}

$\mathrm{C} \uparrow=$ Convincing increase risk; $\mathrm{C} \downarrow=$ convincing decrease risk; $\mathrm{C}-\mathrm{NR}=$ convincing, no relation; $\mathrm{P} \uparrow=$ probable increase risk; $\mathrm{P} \downarrow=$ probable decrease risk; $\mathrm{P}-\mathrm{NR}=$ probable no relation. strongly related to dietary patterns, such as a vegetarian or Mediterranean diet, which are less influenced by misclassification. The null results probably reflect the unreliability of the evidence on dietary fats from cohort studies that differs markedly from the reliability of ecological studies or metabolic ward studies of diet and cholesterol.

One of the exceptions in the body of evidence from prospective cohort studies is n-3 LCPUFA intake or fish consumption and risk of fatal CHD. The evidence is comprehensive in number of studies, duration of follow-up, number of participants and CHD events, geographic location of study populations, homogeneity of association between trials and absence of evidence for publication bias. The observational evidence is convincing that a strong inverse association exists between n-3 LCPUFA or fish intake and risk of CHD. The evidence from randomised controlled trials is concordant, particularly when 2 trials with methodological concerns [Singh et al., 1997; Burr et al., 2003], are excluded from consideration, however, it rests almost entirely on the results from 2 trials (GISSI-P [GISSI-Prevenzione Investigators, 1999], and DART I [Burr et al., 1989]). 


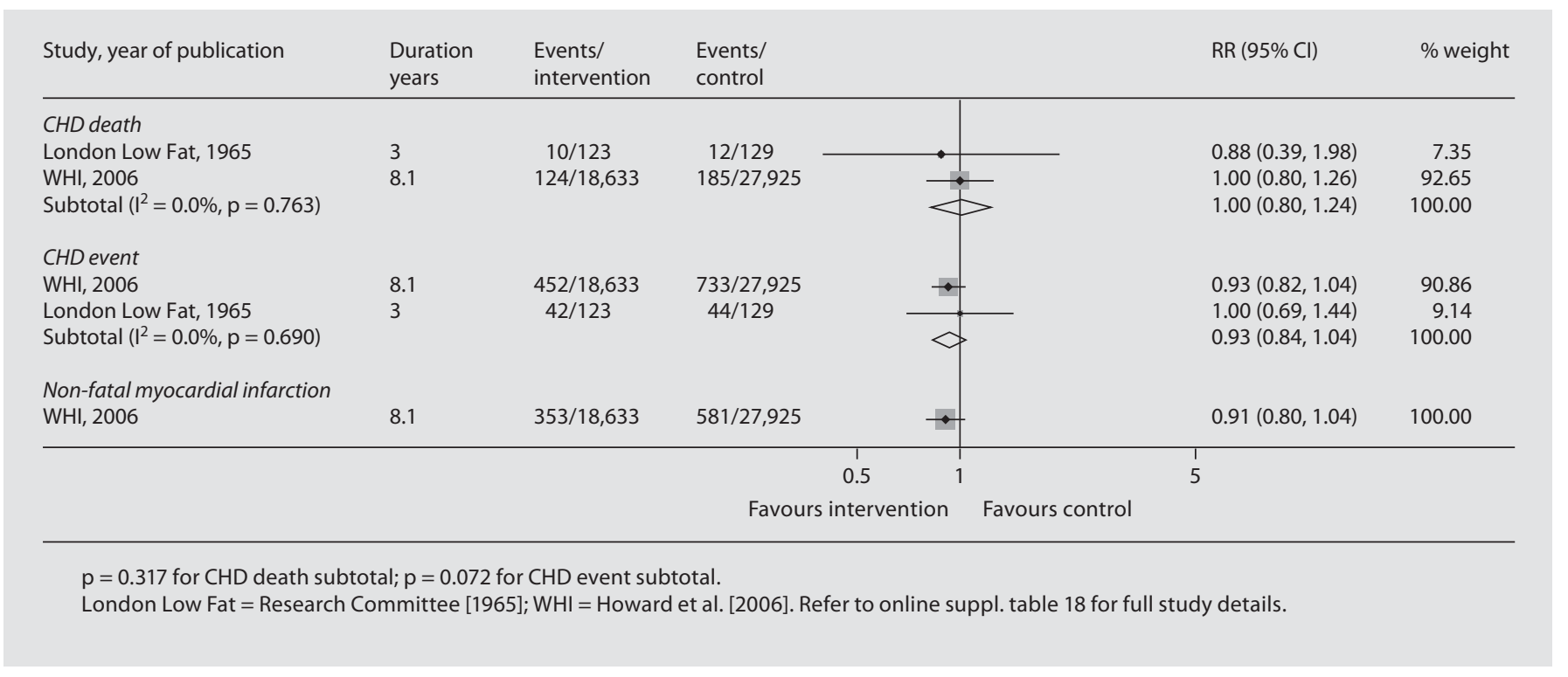

Fig. 21. Meta-analysis of low-fat trials and CHD event.

The observational evidence that TFA are independently associated with increased risk of CHD events is convincing, though based on a more limited body of evidence. The evidence of an association with fatal CHD is not as comprehensive. In view of the consistency and strength of the observational evidence, the absence of evidence from randomised controlled trials should not preclude a convincing judgement.

There is probably no direct relation between total fat intake and risk of CHD. The strongest evidence in support of this judgement comes from the Women's Health Initiative that showed that $\mathrm{CHD}$ risk was not reduced after 8 years of a low-fat diet. The observational evidence, summarised in the meta-analysis, showed no association between total fat intake and CHD risk, although there was heterogeneity between the study results.

Table 4 summarises the strength of evidence of a link between dietary fat and CHD.

The body of evidence from clinical trials of fat-modified diets - excluding n-3 LCPUFA and fish interventions - is limited. The 10 or so published trials are heterogenous in the nature of the dietary intervention and many of the trials have only a small number of CHD deaths or events; nevertheless, taken together, there were slightly more than $600 \mathrm{CHD}$ deaths and 3,700 CHD events in the intervention trials. The heterogeneous nature of the interventions and lack of compliance may undermine the validity of the summary estimates of risk obtained through meta-analysis of the trial results, as does the small number of trials. Several limitations have been well described [Truswell, 2005] but the use of meta-analysis helps to provide consistent display of all the available evidence together with a summary measure of the overall effects.

Clinical trials of fat-modified diets, in particular lowfat or high $\mathrm{P} / \mathrm{S}$ diets, and coronary disease are rarely single factor interventions. Substitution of 1 type of fat for another or reducing total fat intake, invariably results in a range of food substitutions such that intake of other macro- and micronutrients is altered. Many of the early fat intervention trials of CHD required participants to follow a diet lower in cholesterol but with a higher P/S ratio - without a reduction in total fat intake. The results of trials of dietary advice differ from the more reliable evidence from metabolic ward studies. The results of metabolic ward studies [Hegsted et al., 1965; Keys et al., 1965] showed that change in serum cholesterol concentrations could be predicted based on the PUFA, SFA and cholesterol content of the diet. Furthermore, many trials of advice to modify dietary intake of fat have included 1 or more other elements of dietary and non-dietary advice; examples include advice to increase fibre intake, reduce meat consumption, reduce body weight, stop smoking, reduce salt intake, increase fruit and vegetable consumption, increase physical activity, or reduce alcohol consumption. The multifactorial nature of the dietary interventions and accompanying changes in dietary pat- 


\begin{tabular}{|c|c|c|c|c|c|c|}
\hline Study, year of publication & $\begin{array}{l}\text { Duration } \\
\text { years }\end{array}$ & $\begin{array}{l}\text { Events/ } \\
\text { intervention }\end{array}$ & $\begin{array}{l}\text { Events/ } \\
\text { control }\end{array}$ & & $\mathrm{RR}(95 \% \mathrm{Cl})$ & $\%$ weight \\
\hline \multicolumn{7}{|l|}{ CHD death } \\
\hline STARS, 1992 & 3.25 & $1 / 27$ & $3 / 28$ & $\longleftarrow$ & $0.37(0.04,3.35)$ & 5.72 \\
\hline Oslo, 1966 & 5 & $10 / 206$ & $23 / 206$ & $\cdot$ & $0.46(0.22,0.95)$ & 53.85 \\
\hline Finn Mental Hospital (men), 1979 & 4.3 & $6 / 444$ & $12 / 478$ & 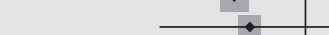 & $0.54(0.21,1.44)$ & 29.47 \\
\hline Finn Mental Hospital (women), 1983 & 3.7 & $3 / 372$ & $3 / 341$ & $\longrightarrow$ & $0.92(0.19,4.51)$ & 10.95 \\
\hline Subtotal $\left(I^{2}=0.0 \%, p=0.874\right)$ & & & & $\longrightarrow$ & $0.52(0.30,0.87)$ & 100.00 \\
\hline \multicolumn{7}{|l|}{ CHD event } \\
\hline Finn Mental Hospital (men), 1979 & 4.3 & $8 / 444$ & $26 / 478$ & $\longrightarrow$ & $0.34(0.16,0.75)$ & 12.81 \\
\hline Finn Mental Hospital (women), 1983 & 3.7 & $3 / 372$ & $8 / 341$ & & $0.35(0.09,1.30)$ & 5.50 \\
\hline STARS, 1992 & 3.25 & $3 / 27$ & $9 / 28$ & 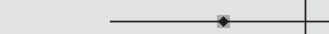 & $0.41(0.12,1.39)$ & 6.35 \\
\hline Oslo, 1966 & 5 & $34 / 206$ & $54 / 206$ & $\rightarrow$ & $0.68(0.46,1.01)$ & 27.86 \\
\hline Veterans' Administration Trial, 1969 & 3.7 & $52 / 424$ & $65 / 422$ & $\rightarrow$ & $0.82(0.58,1.15)$ & 30.72 \\
\hline London Corn and Olive, 1965 & 2 & $15 / 28$ & $11 / 26$ & & $1.17(0.62,2.23)$ & 16.75 \\
\hline Subtotal $\left(1^{2}=40.3 \%, p=0.137\right)$ & & & & $\infty$ & $0.68(0.49,0.94)$ & 100.00 \\
\hline \multicolumn{7}{|l|}{ Total mortality } \\
\hline STARS, 1992 & 3.25 & $1 / 27$ & $3 / 28$ & & $0.37(0.04,3.35)$ & 0.45 \\
\hline Finn Mental Hospital (women), 1983 & 3.7 & $415 / 3,598$ & $465 / 2,836$ & 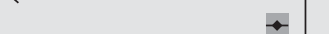 & $0.73(0.65,0.83)$ & 33.97 \\
\hline Finn Mental Hospital (men), 1979 & 4.3 & $287 / 2,276$ & $217 / 1,902$ & $\rightarrow$ & $0.74(0.62,0.89)$ & 26.12 \\
\hline Oslo Diet-Heart Study & 5 & $41 / 206$ & $55 / 206$ & $\rightarrow-$ & $0.79(0.55,1.13)$ & 12.05 \\
\hline Veterans' Administration Trial, 1969 & 3.7 & $174 / 424$ & $177 / 422$ & 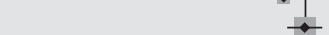 & $0.98(0.83,1.17)$ & 27.40 \\
\hline \multirow[t]{3}{*}{ Subtotal $\left(I^{2}=52.0 \%, p=0.080\right)$} & & & & $\diamond$ & $0.80(0.69,0.93)$ & 100.00 \\
\hline & & & & 0.5 & $\begin{array}{l}1 \\
5\end{array}$ & \\
\hline & & & & Favours intervention & Favours control & \\
\hline
\end{tabular}

$p=0.014$ for $C H D$ death subtotal; $p=0.020$ for CHD event subtotal; $p=0.004$ for total mortality subtotal.

STARS = Watts et al. [1992]; Oslo Diet-Heart Study = Leren [1970]; Finnish Mental Hospital = Turpeinen [1979]; Veterans' Administration Trial = Dayton and Pearce [1969]; London Corn and Olive $=$ Rose et al. [1965]. Refer to online suppl. table 18 for full study details.

Fig. 22. Meta-analysis of PUFA - SFA modified trials including studies showing change in serum cholesterol concentrations with intervention.

\begin{tabular}{|c|c|c|c|c|c|c|c|}
\hline Author, year & Duration & $\begin{array}{l}\text { Events/ } \\
\text { intervention }\end{array}$ & $\begin{array}{l}\text { Events/ } \\
\text { control }\end{array}$ & & & $\mathrm{RR}(95 \% \mathrm{Cl})$ & $\%$ weight \\
\hline Brox et al., 2001 & 14 months & $0 / 80$ & $1 / 40$ & $\leftarrow$ & & $0.17(0.01,4.15)$ & 0.08 \\
\hline Leaf et al., 1991 & 6 months & $0 / 275$ & $2 / 276$ & & & $0.20(0.01,4.19)$ & 0.08 \\
\hline Kaul et al., 1992 & 6 months & $0 / 58$ & $1 / 49$ & & & $0.29(0.01,6.92)$ & 0.08 \\
\hline Sacks et al., 1995 & 28 months & $0 / 31$ & $1 / 28$ & $\rightarrow$ & & $0.31(0.01,7.38)$ & 0.08 \\
\hline Johansen et al., 1999 & 6.5 months & $1 / 250$ & $3 / 250$ & . & - & $0.34(0.04,3.21)$ & 0.15 \\
\hline Raitt et al., 2005 & 23 months & $4 / 100$ & $10 / 100$ & $\rightarrow$ & - & $0.42(0.14,1.31)$ & 0.59 \\
\hline von Schacky et al., 1999 & 1 year & $1 / 112$ & $2 / 111$ &. & & $0.50(0.05,5.44)$ & 0.13 \\
\hline Brouwer et al., 2006 & 1 year & $8 / 273$ & $14 / 273$ & $\longrightarrow$ & - & $0.58(0.25,1.37)$ & 1.03 \\
\hline Burr et al., 1989 & 1 year & $94 / 1,015$ & $130 / 1,018$ & |نه & & $0.75(0.58,0.96)$ & 9.20 \\
\hline GISSI-P, 1999 & 2 months & $472 / 5,666$ & $545 / 5,668$ & 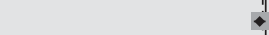 & & $0.88(0.78,0.99)$ & 22.66 \\
\hline GISSI-HF, 2008 & 3.9 years & $955 / 3,494$ & $1,014 / 3,481$ & & & $0.95(0.88,1.03)$ & 29.48 \\
\hline Nilsen et al., 2001 & 24 months & $11 / 150$ & $11 / 150$ & & - & $1.00(0.45,2.24)$ & 1.15 \\
\hline Yokoyama et al., 2007 & 5 years & $286 / 9,326$ & $265 / 9,316$ & & . & $1.08(0.91,1.27)$ & 16.32 \\
\hline Leaf et al., 2005 & 1 year & $13 / 200$ & $12 / 202$ & & & $1.09(0.51,2.33)$ & 1.28 \\
\hline Burr et al., 2005 & 3.9 years & $283 / 1,571$ & $242 / 1,543$ & & 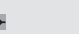 & $1.13(0.96,1.32)$ & 17.01 \\
\hline Erstland et al., 1996 & 1 year & $8 / 322$ & $6 / 293$ & & 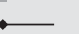 & $1.21(0.42,3.44)$ & 0.69 \\
\hline \multirow[t]{3}{*}{ Overall $\left(I^{2}=23.4 \%, p=0.188\right)$} & & & & & & $0.95(0.87,1.03)$ & 100.00 \\
\hline & & & \multicolumn{5}{|c|}{$0.51 \quad 5$} \\
\hline & & & \multicolumn{2}{|r|}{ Favours intervention } & \multicolumn{2}{|c|}{ Favours control } & \\
\hline
\end{tabular}

Fig. 23. Meta-analysis of fish or n-3 LCPUFA trials and total mortality, including DART II. 


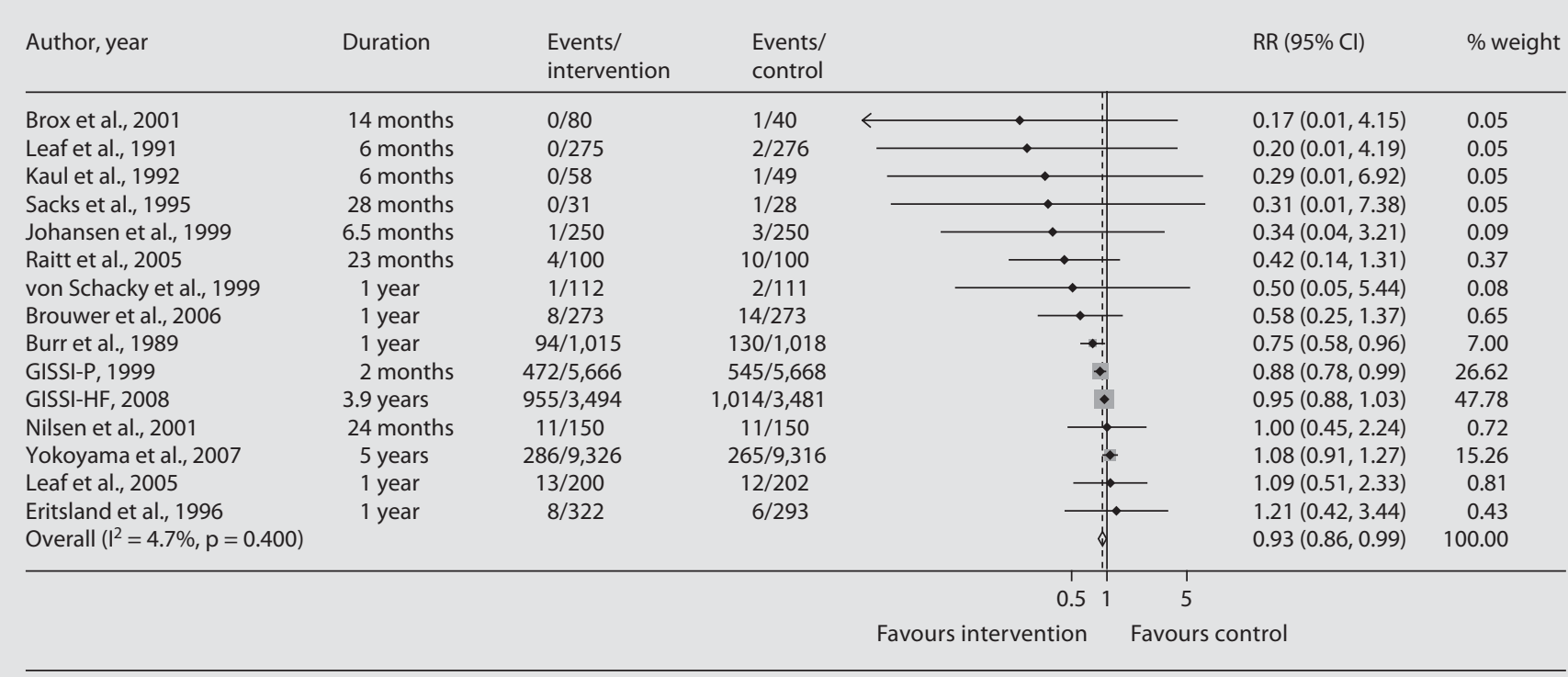

$p=0.027$ overall.

Fig. 24. Meta-analysis of fish or n-3 LCPUFA trials and total mortality, excluding DART II.

terns makes it difficult to disentangle the specific effects of dietary fat from other components of the diet. In effect, the dietary interventions are not homogeneous, and are unreliable. However, in trials of dietary advice to modify intake of dietary lipids where the change in fat intake or in the $\mathrm{P} / \mathrm{S}$ ratio has been large, and there is good evidence of participant compliance, a meta-analysis of trials, which together provide a large number of endpoint events, should provide more reliable evidence.

In this regard, the meta-analysis of trials in which serum cholesterol concentrations in the high $\mathrm{P} / \mathrm{S}$ diet group were significantly lower than in the control group, revealed that a diet higher in PUFA and lower in SFA decreased the risk of fatal CHD; however, this was significant only after inclusion of results from the Oslo study which included smoking cessation as part of the treatment. The cholesterol-lowering effect of the high P/S diet is driven largely by the reduction in SFA intake as shown in the metabolic ward studies [Clarke et al., 1997]. The evidence from metabolic ward studies clearly shows that diets low in SFA reduce total cholesterol and should therefore reduce the risk of CHD. However, the meta-analysis of results from cohort studies - albeit from a limited number of studies - showed no association between SFA intake and $\mathrm{CHD}$, demonstrating their unreliability.
The observational evidence for an association between dietary PUFA and CHD risk is inconsistent and is unreliable. The summary estimate from the meta-analysis showed a significant increase in the RR of CHD death in the highest category of dietary PUFA (RR 1.25, 95\% CI $1.06-1.47, \mathrm{p}=0.009$ ) in contrast, a 5\% increase in PUFA intake was associated with a significant reduction in CHD events (RR 0.84, 95\% CI 0.70-1.00, $\mathrm{p}=0.049$ ).

The observational evidence for dietary MUFA shows no association with CHD risk.

Clinical trials of n-3 LCPUFA and CHD are better suited to meta-analysis inasmuch as most interventions are single factor, involving consumption of a fish oil or n-3 LCPUFA rich purified oil supplement. However, treatment effects may be modified by the amount and proportions of n-3 LCPUFA consumed during treatment, by the food or supplement form of the LCPUFA, the absolute risk of CHD in the study population, the duration of follow-up, or whether the trial was to prevent recurrence or occurrence of CHD. Several meta-analyses of cohort studies and randomised controlled trials have been published. The meta-analysis by Hooper et al. [2006] was conducted according to the conventions for systematic reviews developed by the Cochrane Collaboration and reviewed the evidence for an effect of $n-3$ fatty acids on cardiovascular events. The authors limited their re- 


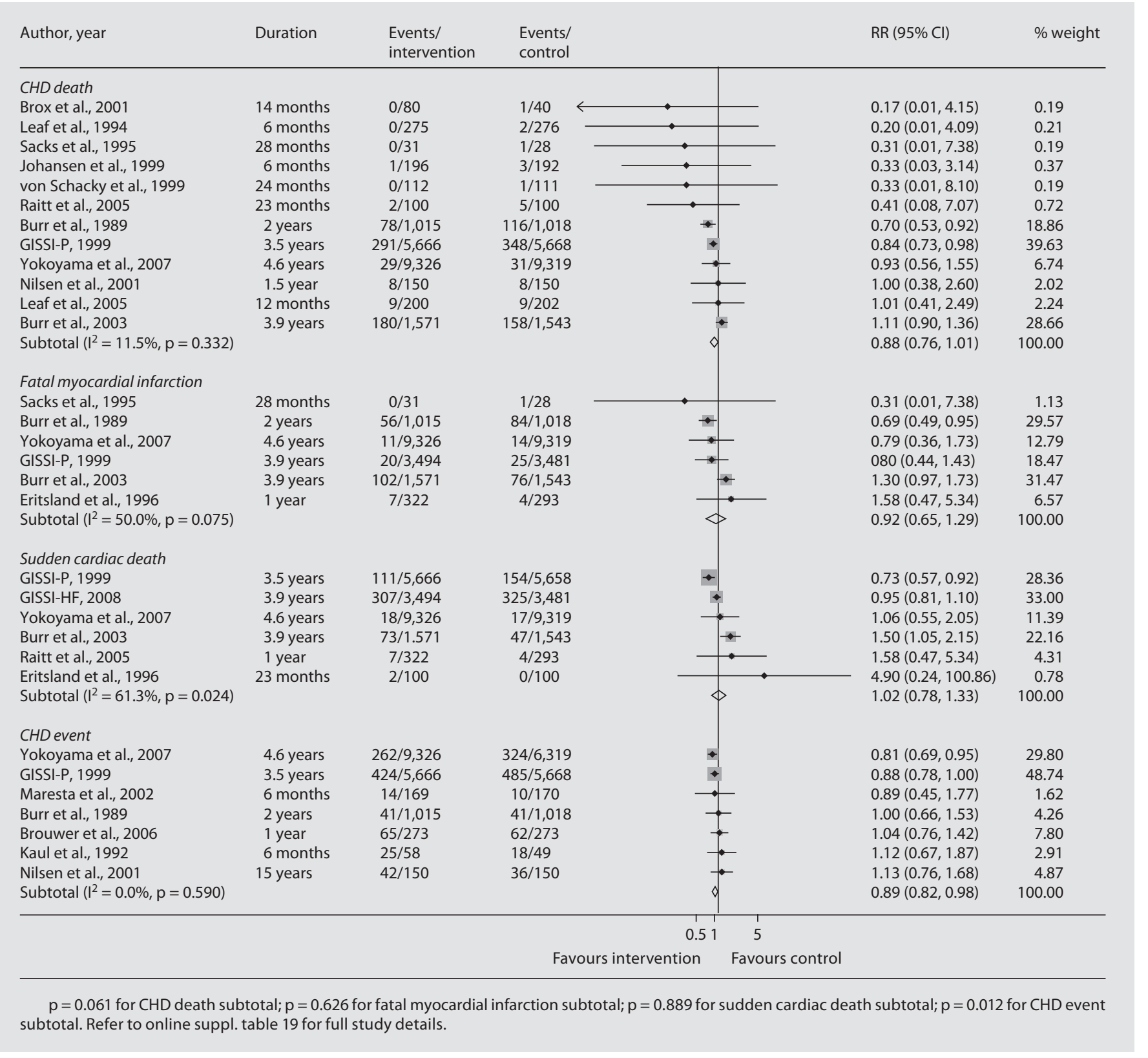

Fig. 25. Meta-analysis of fish or n-3 LCPUFA trials and CHD fatal events, including DART II.

view to studies in which an estimate of n-3 fatty acid intake could be verified because their primary hypothesis was to test the effect of 'long or shorter chain' n-3 fatty acids. Thus, relevant studies were excluded in which exposure to n-3 LCPUFA was assessed by fatty acid biomarkers or in which fish consumption but not $n-3$ LCPUFA intake was measured. The overall conclusion was an absence of a clear effect of n-3 PUFA on total mortality (RR $0.87,95 \%$ CI $0.73-1.03$ ) or combined cardiovas- cular events (RR 0.95, 95\% CI 0.82-1.12). A number of other critical points have been raised about the systematic review by Hooper et al., to which the authors have given considered and substantiated responses [Twisselmann, 2006].

The meta-analysis by Mozaffarian and Rimm [2006] on n-3 LCPUFA and risk of CHD mortality combined the results from cohort and randomised controlled trials to conclude that 1-2 servings per week of fish reduces the 


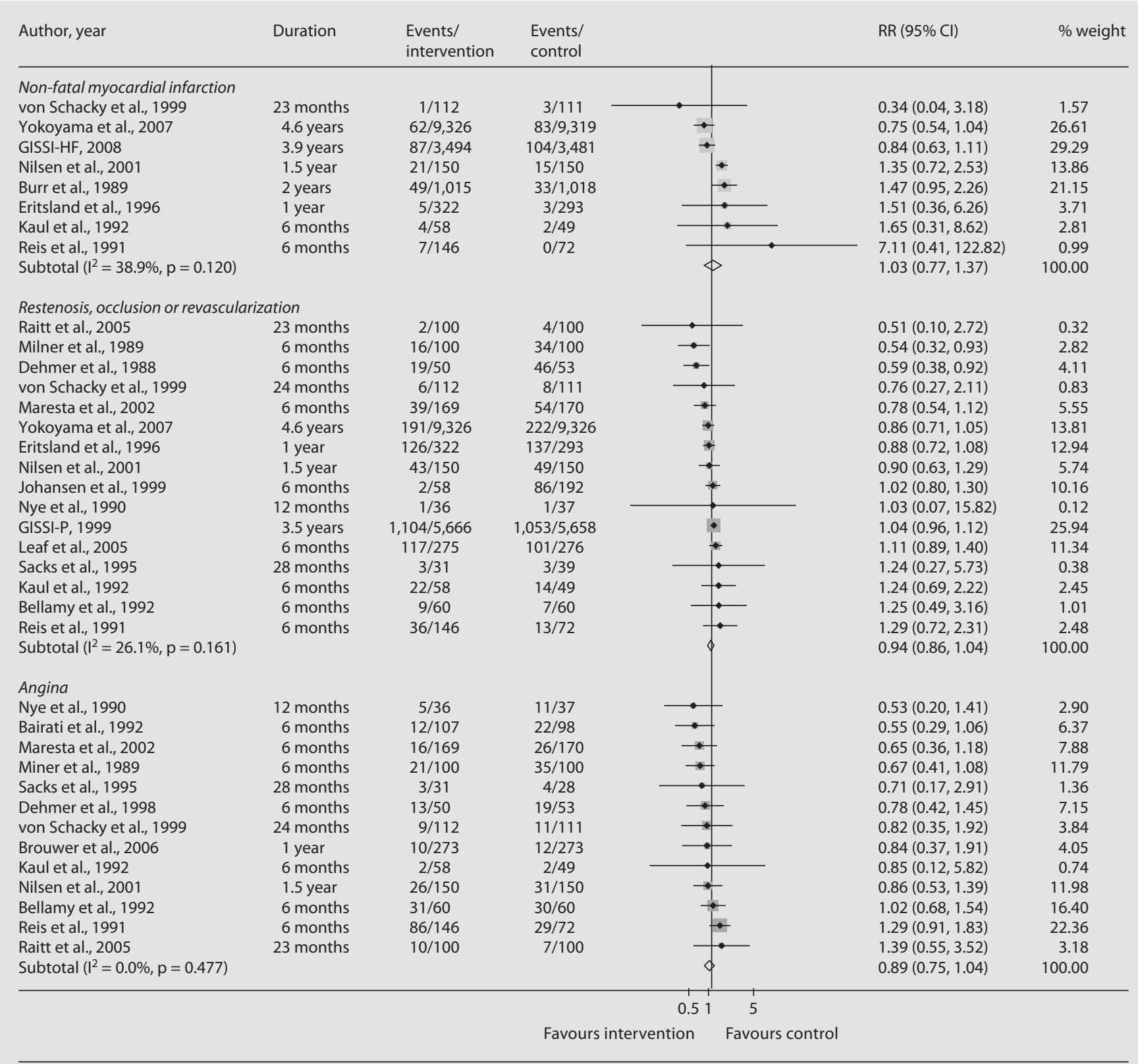

$p=0.864$ for non-fatal myocardial infarction subtotal; $p=0.211$ for restenosis, occlusion or revascularization subtotal; $p=0.149$ for angina subtotal. Refer to online suppl. table 19 for full study details.

Fig. 26. Meta-analysis of fish or n-3 LCPUFA trials and CHD non-fatal events.

risk of coronary death by $36 \%$ (95\% CI $20-50 \%)$. The risk reduction in total mortality with fish consumption was $17 \%$ (95\% CI 0-32\%). This estimate was calculated using conventional meta-analysis and is quite similar in magnitude to that reported by Hooper et al. [2006] though the CIs are different.

Dietary Fat and Coronary Heart Disease
We have updated the meta-analyses with studies published since the work of Hooper et al. [2006] and Mozaffarian and Rimm [2006]. The totality of evidence from observational cohort studies consistently shows that high intake of n-3 LCPUFA or consumption of fish is associated with significantly lower risk of fatal and non-fatal 


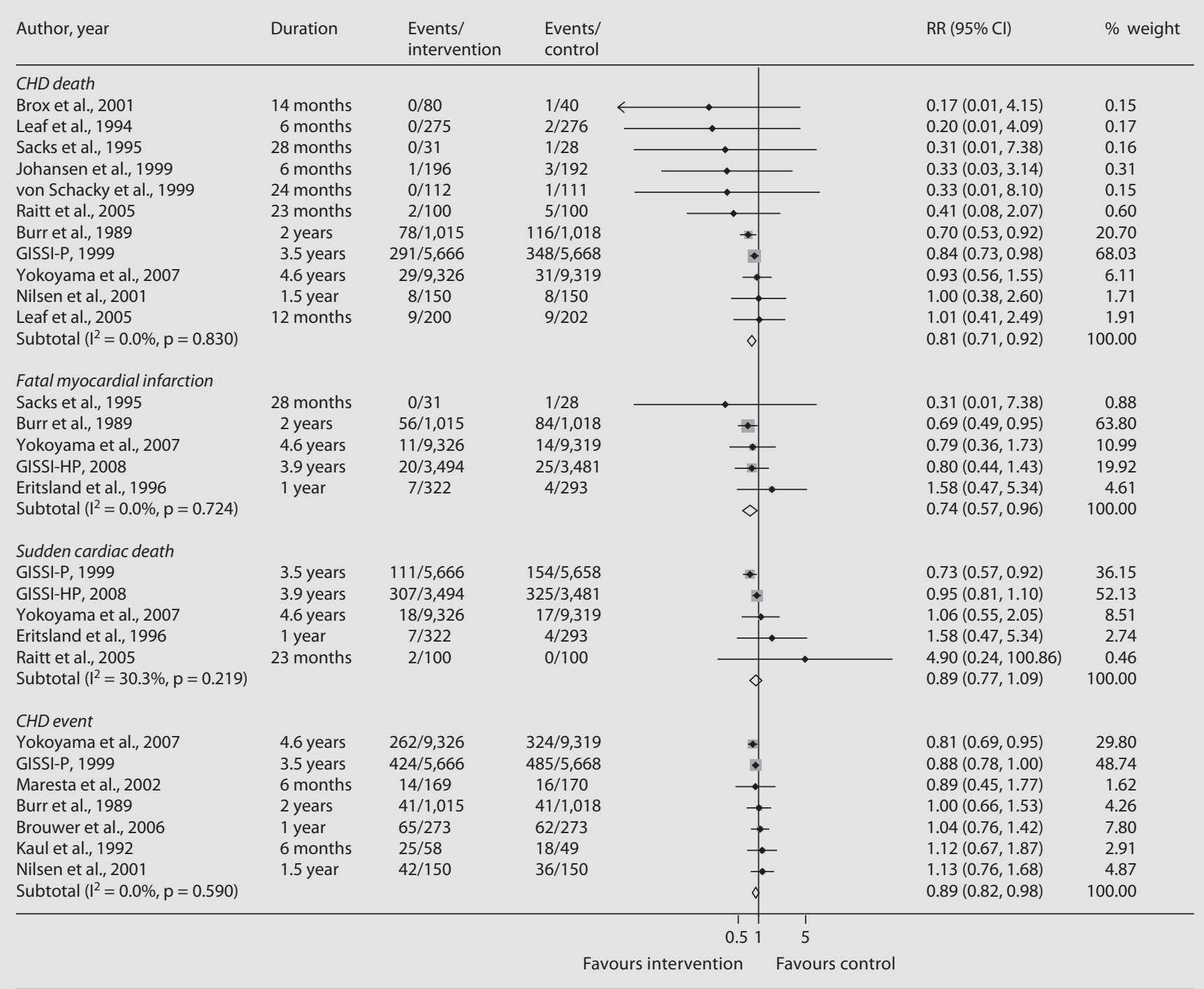

$p=0.001$ for $\mathrm{CHD}$ death subtotal; $\mathrm{p}=0.025$ for fatal myocardial infarction subtotal; $\mathrm{p}=0.251$ for sudden cardiac death subtotal; $\mathrm{p}=0.012$ for $\mathrm{CHD}$ event subtotal. Refer to online suppl. table 19 for full study details.

Fig. 27. Meta-analysis of fish or n-3 LCPUFA trials and CHD fatal events, excluding DART II.

CHD as well as combined CHD event. On the other hand, the results of randomised controlled trials, particularly in relation to fatal CHD, fatal MI or sudden cardiac death, do not show a beneficial effect of n-3 LCPUFA, though they do reveal a significant reduction in risk of total CHD events with treatment. To be convinced that $n-3$ LCPUFA decreases the risk of CHD it is desirable to find concordance of results between observational and cohort studies. The absence of concordance does not preclude a convincing judgement inasmuch as the limitations of study design inherent in cohort or intervention studies may account for some of the discrepancy. One of the most obvious differences between the cohort and intervention trials is the markedly longer duration of follow-up in the cohort studies, 17 years compared with 2 years for CHD death. It is also possible that fish consumers in Europe and North American have a 'healthy' lifestyle, and the inverse association between n-3 LCPUFA intake and fish consumption may be explained by residual confounding. Participants in the intervention trials were generally older at recruitment and at higher initial risk of CHD. Exclusion from the meta-analysis of 1 intervention trial (DART 
II) with methodological concerns substantially altered the significance of the summary estimates of RR such that fatal CHD, fatal MI, sudden cardiac death were significantly reduced by n-3 LCPUFA; furthermore, the results of the remaining studies showed no heterogeneity. In the absence of DART II, the GISSI-P and DART I trials contribute $90 \%$ of the fatal CHD events on which the summary estimate is based. Thus, the evidence from randomised controlled trials about the protective effects of n-3 LCPUFA on fatal CHD rests on the results of 2 trials, both of which have some methodological limitations which may have introduced bias.

\section{Post-Script}

A pooled analysis of 11 cohort studies of dietary fat and coronary disease was presented to the Expert Consultation (Nov, 2008) and the manuscript was published shortly thereafter in May 2009 [Jakobsen et al., 2009]. In the judgement of the Expert Consultation, the results of the 'Pooling Project of Cohort Studies on Diet and Coronary Disease' were a significant advance in quality on the update, undertaken by the Consultation, of the published meta-analyses of observational trials. The Pooling Project combined the results from 11 cohort studies - each meeting criteria for quality of dietary assessment, years of follow-up, and ascertainment of events - to examine the effect on CHD death and CHD events of replacing SFA with MUFA, PUFA or carbohydrate. The main finding was a significantly decreased risk of CHD death and CHD events when PUFA replaces SFA. The multivariate-adjusted hazard ratio for CHD death per 5\% TE incremental substitution of PUFA for SFA was 0.87 (95\% CI 0.77-0.97); for CHD events, the hazard ratio for the same fat substitution was 0.74 (95\% CI 0.61-0.89). This result from the pooling of observational studies, along with supportive evidence from clinical trials of lower CHD risk in high $\mathrm{P} / \mathrm{S}$ diets, and the effects of PUFA to lower LDL cholesterol and the total:high-density lipoprotein ratio, led the Consultation to conclude there was convincing evidence of lower CHD risk when PUFA replaces SFA.

\section{Disclosure Statement}

Ms. Miller has nothing to declare. Dr. Skeaff has conducted clinical research trials which have been funded through the University by Unilever and Fonterra. He has served on governmental and non-governmental advisory groups.

\section{References}

Albert CM, Campos H, et al: Blood levels of longchain $n-3$ fatty acids and the risk of sudden death. New Engl J Med 2002;346:1113-1118.

- Albert CM, Hennekens CH, et al: Fish consumption and risk of sudden cardiac death. JAMA 1998;279:23-28.

-Ascherio A, Rimm EB, et al: Dietary intake of marine $\mathrm{n}-3$ fatty acids, fish intake, and the risk of coronary disease among men. New Engl J Med 1995;332:977-982.

-Ascherio A, Rimm EB, et al: Dietary fat and risk of coronary heart disease in men: cohort follow up study in the United States. BMJ 1996; 313:84-90.

Bairati I, Roy L, Meyer F: Double-blind, randomized, controlled trial of fish oil supplements in prevention of recurrence of stenosis after coronary angioplasty. Circulation 1992;85:950-956.

-Bang HO, Dyerberg J, et al: The composition of food consumed by Greenland Eskimos. Acta Med Scand 1976;200:69-73.

- Begg CB, Mazumdar M: Operating characteristics of a rank correlation test for publication bias. Biometrics 1994;50:1088-1101.

- Bellamy CM, Schofield PM, Faragher EB, et al: Can supplementation of diet with omega-3 polyunsaturated fatty acids reduce coronary angioplasty restenosis rate? Eur Heart J 1992; 13:1626-1631.

Bemelmans WJ, Broer J, et al: Effect of an increased intake of alpha-linolenic acid and group nutritional education on cardiovascular risk factors: the Mediterranean Alphalinolenic Enriched Groningen Dietary Intervention (MARGARIN) study. Am J Clin Nutr 2002;75:221-227.

Black HS, Herd JA, et al: Effect of a low-fat diet on the incidence of actinic keratosis. New Engl J Med 1994;330:1272-1275.

Blacket RB, Leelarthaepin B, et al: The synergistic effect of weight loss and changes in dietary lipids on the serum cholesterol of obese men with hypercholesterolaemia: implications for prevention of coronary heart disease. Aust NZ J Med 1979;9:521-529.

Boniface DR, Tefft ME: Dietary fats and 16-year coronary heart disease mortality in a cohort of men and women in Great Britain. Eur J Clin Nutr 2002;56:786-792.

Booker CS, Mann JI: Trans fatty acids and cardiovascular health: translation of the evidence base. Nutr Metab Cardiovasc Dis 2008;18:448-456.
Borchgrevink CF, Skaga E, et al: Absence of prophylactic effect of linolenic acid in patients with coronary heart-disease. Lancet 1966;2: 187-189.

Brouwer IA, Katan MB, et al: Dietary alpha-linolenic acid is associated with reduced risk of fatal coronary heart disease, but increased prostate cancer risk: a meta-analysis. J Nutr 2004;134:919-922.

Brouwer IA, Zock PL, et al: Effect of fish oil on ventricular tachyarrhythmia and death in patients with implantable cardioverter defibrillators: the Study on Omega-3 Fatty Acids and Ventricular Arrhythmia (SOFA) randomized trial. JAMA 2006;295:2613-2619.

-Brox J, Olaussen K, Osterud B, et al: A long-term seal- and cod-liver-oil supplementation in hypercholesterolemic subjects. Lipids 2001; 36:7-13.

Bucher HC, Hengstler P, et al: n-3 polyunsaturated fatty acids in coronary heart disease: a meta-analysis of randomized controlled trials. Am J Med 2002;112:298-304.

Burr ML, Ashfield-Watt PA, et al: Lack of benefit of dietary advice to men with angina: results of a controlled trial. Eur J Clin Nutr 2003;57: 193-200. 
- Burr ML, Fehily AM, et al: Effects of changes in fat, fish, and fibre intakes on death and myocardial reinfarction: diet and reinfarction trial (DART). Lancet 1989;2:757-761.

-Clarke R, Frost C, et al: Dietary lipids and blood cholesterol: quantitative meta-analysis of metabolic ward studies. BMJ 1997;314:112117.

Daviglus ML, Stamler J, et al: Fish consumption and the 30-year risk of fatal myocardial infarction. New Engl J Med 1997;336:10461053.

Dayton S, Pearce ML: Prevention of coronary heart disease and other complications of arteriosclerosis by modified diet. Am J Med 1969;46:751-762.

-Dehmer GJ, Popma JJ, van den Berg EK, et al: Reduction in the rate of early restenosis after coronary angioplasty by a diet supplemented with n-3 fatty acids. N Engl J Med 1988;319: 733-740.

$\checkmark$ de Lorgeril M, Renaud S, et al: Mediterranean alpha-linolenic acid-rich diet in secondary prevention of coronary heart disease. Lancet 1994;343:1454-1459. Erratum in Lancet 1995;345:738.

Dyerberg J, Bang H: Haemostatic function and platelet polyunsaturated fatty acids in Eskimos. Lancet 1979;2:433-435.

Dyerberg J, Bang HO, et al: Eicosapentaenoic acid and prevention of thrombosis and atherosclerosis. Lancet 1978;2:2:117-119.

Eritsland J, Arnesen H, Gronseth K, et al: Effect of dietary supplementation with n-3 fatty acids on coronary artery bypass graft patency. Am J Cardiol 1996;77:31-36.

Erkkila A, de Mello V, et al: Dietary fatty acids and cardiovascular disease: an epidemiological approach. Prog Lipid Res 2008;47:172187.

Erkkila AT, Lehto S, et al: n-3 fatty acids and 5year risks of death and cardiovascular disease events in patients with coronary artery disease. Am J Clin Nutr 2003;78:65-71.

-Esrey KL, Joseph L, et al: Relationship between dietary intake and coronary heart disease mortality: Lipid Research Clinics Prevalence Follow-up Study. J Clin Epidemiol 1996;49: 211-216.

Expression of concern. BMJ 2005;331:266.

Folsom AR, Demissie Z: Fish intake, marine omega-3 fatty acids, and mortality in a cohort of postmenopausal women. Am J Epidemiol 2004;160:1005-1010.

Frantz ID Jr, Dawson EA, et al: Test of effect of lipid lowering by diet on cardiovascular risk: the Minnesota Coronary Survey. Arteriosclerosis 1989;9:129-135.

Fraser GE, Strahan TM, et al: Effects of traditional coronary risk factors on rates of incident coronary events in a low-risk population: the Adventist Health Study. Circulation 1992;86:406-413.
GISSI-HF Investigators: Effect of $n-3$ polyunsaturated fatty acids in patients with chronic heart failure (the GISSI-HF trial): a randomised, double-blind, placebo-controlled trial. Lancet 2008;372:1223-1230.

GISSI-Prevenzione Investigators: Dietary supplementation with $n-3$ polyunsaturated fatty acids and vitamin $\mathrm{E}$ after myocardial infarction: results of the GISSI-Prevenzione trial. Lancet 1999;354:447-455.

He K, Song Y, et al: Accumulated evidence on fish consumption and coronary heart disease mortality: a meta-analysis of cohort studies. Circulation 2004;109:2705-2711.

Hegsted DM, McGandy RB, et al: Quantitative effects of dietary fat on serum cholesterol in man. Am J Clin Nutr 1965;17:281-295.

Hooper L, Griffiths E, et al: Dietetic guidelines: diet in secondary prevention of cardiovascular disease (first update, June 2003). J Hum Nutr Dietetics 2004b;17:337-349.

Hooper L, Harrison RA, Summerbell CD, et al.: Omega 3 fatty acids for prevention and treatment of cardiovascular disease. Cochrane Database of Sys Rev 2004a;CD003177.

-Hooper L, Summerbell CD, et al: Dietary fat intake and prevention of cardiovascular disease: systematic review. BMJ 2001;322:757763.

Hooper L, Summerbell CD, Higgins JP, et al: Reduced or modified dietary fat for preventing cardiovascular disease. [update of Cochrane Database Syst Rev 2000;CD002137; PMID: 10796866]. Cochrane Database of Sys Rev 2001;CD002137.

Hooper L, Thompson RL, et al: Risks and benefits of omega-3 fats for mortality, cardiovascular disease, and cancer: systematic review. BMJ 2006;332:752-760.

- Howard BV, Van Horn L, et al: Low-fat dietary pattern and risk of cardiovascular disease: the Women's Health Initiative Randomized Controlled Dietary Modification Trial. JAMA 2006;295:655-666.

Hu FB, Bronner L, et al: Fish and omega-3 fatty acid intake and risk of coronary heart disease in women. JAMA 2002;287:1815-1821.

Hu FB, Stampfer MJ, et al: Dietary fat intake and the risk of coronary heart disease in women. New Engl J Med 1997;337:1491-1499.

- Hu FB, Stampfer MJ, et al: Dietary intake of alpha-linolenic acid and risk of fatal ischemic heart disease among women. Am J Clin Nutr 1999;69:890-897.

Iso H, Kobayashi M, et al: Intake of fish and n-3 fatty acids and risk of coronary heart disease among Japanese: the Japan Public Health Center-Based (JPHC) Study Cohort I. Circulation 2006;113:195-202.

Jakobsen MU, O'Reilly EJ, et al: Major types of dietary fat and risk of coronary heart disease: a pooled analysis of 11 cohort studies. Am J Clin Nutr 2009;89:1425-1432.
Jakobsen MU, Overvad K, et al: Dietary fat and risk of coronary heart disease: possible effect modification by gender and age. Am J Epidemiol 2004;160:141-149.

-Jarvinen R, Knekt P, et al: Intake of fish and longchain $n-3$ fatty acids and the risk of coronary heart mortality in men and women. Br J Nutr 2006;95:824-829.

Jenkins DJ, Josse AR, et al: Fish-oil supplementation in patients with implantable cardioverter defibrillators: a meta-analysis. CMAJ 2008;178:157-164.

Johansen O, Brekke M, Seljeflot I, et al: N-3 fatty acids do not prevent restenosis after coronary angioplasty: results from the CART study. Coronary Angioplasty Restenosis Trial. J Am Coll Cardiol 1999;33:1619-1626.

Kaul U, Sanghvi S, Bahl VK, et al: Fish oil supplements for prevention of restenosis after coronary angioplasty. Int J Cardiol 1992;35: 87-93.

Keys A: Coronary heart disease in seven countries. Circulation 1980;41:1-211.

Keys A, Anderson J, et al: Serum cholesterol response to changes in the diet. IV. Particular saturated fatty acids in the diet. Metabolism 1965; 14:776-787.

Keys A, Menotti A, et al: The diet and 15-year death rate in the Seven Countries Study. Am J Epidemiol 1986;124:903-915.

Kromhout D, Bosschieter EB, et al: The inverse relation between fish consumption and 20 year mortality from coronary heart disease. New Engl J Med 1985;312:1205-1209.

Kromhout D, Feskens EJM, et al: The protective effect of a small amount of fish on coronary heart disease mortality in an elderly population. Int J Epidemiol 1995a;24:340-345.

Kromhout D, Menotti A, et al: Dietary saturated and trans fatty acids and cholesterol and 25year mortality from coronary heart disease: the Seven Countries Study. Prev Med 1995b;24:308-315.

Leaf A: Dietary prevention of coronary heart disease: the Lyon Diet Heart Study. Circulation 1999;99:733-735.

Leaf A, Albert CM, et al: Prevention of fatal arrhythmias in high-risk subjects by fish oil n-3 fatty acid intake. Circulation 2005; 112 : 2762-2768.

Leaf A, Jorgensen MB, Jacobs AK, et al: Do fish oils prevent restenosis after coronary angioplasty? Circulation 1994;90:2248-2257.

Lemaitre RN, King IB, et al: $n-3$ polyunsaturated fatty acids, fatal ischemic heart disease, and nonfatal myocardial infarction in older adults: the Cardiovascular Health Study. Am J Clin Nutr 2003;77:279-280.

Leren P: The Oslo Diet-Heart Study: eleven-year report. Circulation 1970;42:935-942.

-Mann JI, Appleby PN, et al: Dietary determinants of ischaemic heart disease in health conscious individuals. Heart 1997;78:450-455. 
-Maresta A, Balduccelli M, Varani E, et al: Prevention of postcoronary angioplasty restenosis by omega-3 fatty acids: main results of the Esapent for Prevention of Restenosis ITalian Study (ESPRIT). Am Heart J 2002; 143:E5.

Medical Research Council: Controlled trial of soya-bean oil in myocardial infarction. Lancet 1968;2:693-699.

Miettinen M, Turpeinen O, et al: Effect of cholesterol-lowering diet on mortality from coronary heart-disease and other causes. A twelve-year clinical trial in men and women. Lancet 1972;2:835-838.

Miettinen M, Turpeinen O, et al: Dietary prevention of coronary heart disease in women: the Finnish Mental Hospital Study. Int J Epidemiol 1983;12:17-25.

-Milner MR, Gallino RA, Leffingwell A, et al: Usefulness of fish oil supplements in preventing clinical evidence of restenosis after percutaneous transluminal coronary angioplasty. Am J Cardiol 1989;64:294-299.

Morris MC, Manson JE, Rosner B, et al: Fish consumption and cardiovascular disease in the physicians' health study: a prospective study. Am J Epidemiol 1995;142:166-175.

Mozaffarian D, Ascherio A, Hu FB, et al: Interplay between different polyunsaturated fatty acids and risk of coronary heart disease in men. Circulation 2005;111:157-164.

Mozaffarian D, Katan MB, et al: Trans fatty acids and cardiovascular disease. New Engl J Med 2006;354:1601-1613.

Mozaffarian D, Lemaitre RN, et al: Cardiac benefits of fish consumption may depend on the type of fish meal consumed: the Cardiovascular Health Study. Circulation 2003;107: 1372-1377.

Mozaffarian D, Rimm EB: Fish intake, contaminants, and human health: evaluating the risks and the benefits. JAMA 2006;296: 1885-1899.

- Nakamura Y, Ueshima H, et al: Association between fish consumption and all-cause and cause-specific mortality in Japan: NIPPON DATA80, 1980-99. Am J Med 2005;118:239245.

Natvig H, Borchgrevink CF, et al: A controlled trial of the effect of linolenic acid on incidence of coronary heart disease. The Norwegian vegetable oil experiment of 1965-66. Scand J Clin Lab Invest Suppl 1968;105:120.

Nilsen DWT, Albrektsen G, Landmark K, et al: Effects of a high-dose concentrate of n-3 fatty acids or corn oil introduced early after an acute myocardial infarction on serum triacylglycerol and HDL cholesterol. Am J Clin Nutr 2001;74:50-56.

- Norell SE, Ahlbom A, et al: Fish consumption and mortality from coronary heart disease. Br Med J (Clin Res Ed) 1986;293:426.
Nye ER, Ablett MB, Robertson MC, et al: Effect of eicosapentaenoic acid on restenosis rate, clinical course and blood lipids in patients after percutaneous transluminal coronary angioplasty. Aust N Z J Med 1990;20:549552.

Oh K, Hu FB, et al: Dietary fat intake and risk of coronary heart disease in women: 20 years of follow-up of the Nurses' Health Study. Am J Epidemiol 2005; 161:672-679.

Oomen CM, Feskens EJ, et al: Fish consumption and coronary heart disease mortality in Finland, Italy, and The Netherlands. Am J Epidemiol 2000;151:999-1006.

Oomen CM, Ocké MC, et al: Association between trans fatty acid intake and 10 -year risk of coronary heart disease in the Zutphen Elderly Study: a prospective population-based study. Lancet 2001;357:746-751.

Osler M, Andreasen AH, et al: No inverse association between fish consumption and risk of death from all causes, and incidence of coronary heart disease in middle-aged, Danish adults. J Clin Epidemiol 2003;56:274-279.

- Pietinen P, Ascherio A, et al: Intake of fatty acids and risk of coronary heart disease in a cohort of Finnish men: the Alpha-Tocopherol, BetaCarotene Cancer Prevention Study. Am J Epidemiol 1997;145:876-887.

Posner BM, Cobb JL, et al: Dietary lipid predictors of coronary heart disease in men. The Framingham Study. Arch Intern Med 1991; 151:1181-1187.

Raitt MH, Connor WE, et al: Fish oil supplementation and risk of ventricular tachycardia and ventricular fibrillation in patients with implantable defibrillators: a randomized controlled trial. JAMA 2005;293:2884-2891.

Reis GJ, Kuntz RE, Silverman DI, et al: Effects of serum lipid levels on restenosis after coronary angioplasty. Am J Cardiol 1991;68: 1431-1435.

Research Committee. Low-fat diet in myocardial infarction. A controlled trial. Lancet 1965; 2:501-504.

Rissanen T, Voutilainen S, et al: Fish oil-derived fatty acids, docosahexaenoic acid and docosapentaenoic acid, and the risk of acute coronary events: the Kuopio ischaemic heart disease risk factor study. Circulation 2000; 102:2677-2679.

Rose GA, Thomson WB, et al: Corn oil in treatment of ischaemic heart disease. BMJ 1965;1: 1531-1533.

- Sacks FM, Stone PH, Gibson CM, et al: Controlled trial of fish oil for regression of human coronary atherosclerosis. HARP Research Group. J Am Coll Cardiol 1995;25: 1492-1498.

-Singh RB, Niaz MA, et al: Randomized, doubleblind, placebo-controlled trial of fish oil and mustard oil in patients with suspected acute myocardial infarction: the Indian experiment of infarct survival 4. Cardiovasc Drugs Therapy 1997;11:485-491.
Streppel MT, Ocké MC, et al: Long-term fish consumption and n-3 fatty acid intake in relation to (sudden) coronary heart disease death: the Zutphen study. Eur Heart J 2008; 29:2024-2030.

Truswell AS: Some problems with Cochrane reviews of diet and chronic disease. Eur J Clin Nutr 2005;59:S150-S154.

Tucker KL, Hallfrisch J, et al: The combination of high fruit and vegetable and low saturated fat intakes is more protective against mortality in aging men than is either alone: the Baltimore Longitudinal Study of Aging. J Nutr 2005; 135:556-561.

Turpeinen O: Effect of cholesterol-lowering diet on mortality from coronary heart disease and other causes. Circulation 1979;59:1-7.

- Twisselmann B: Risks and benefits of omega-3 fats: summary of responses. BMJ 2006;332: 915-916.

von Schacky C, Angerer P, Kothny W, et al: The effect of dietary n-3 fatty acids on coronary atherosclerosis. A randomized, placebocontrolled trial. Ann Intern Med 1999;130: 554-562.

Wang C, Harris WS, et al: n-3 Fatty acids from fish or fish-oil supplements, but not alphalinolenic acid, benefit cardiovascular disease outcomes in primary- and secondary-prevention studies: a systematic review. American Journal of Clinical Nutrition 2006;84: 5-17.

Watts GF, Lewis B, et al: Effects on coronary artery disease of lipid-lowering diet, or diet plus cholestyramine, in the St Thomas' Atherosclerosis Regression Study (STARS). Lancet 1992;339:563-569.

Whelton SP, He J, et al: Meta-analysis of observational studies on fish intake and coronary heart disease. Am J Cardiol 2004;93:11191123.

Xu J, Eilat-Adar S, et al: Dietary fat intake and risk of coronary heart disease: the Strong Heart Study. Am J Clin Nutr 2006;84:894902.

- Yamagishi K, Nettleton JA, Folsom AR, et al: Plasma fatty acid composition and incident heart failure in middle-aged adults: the Atherosclerosis Risk in Communities (ARIC) Study. Am Heart J 2008;156:965-974.

- Yokoyama M, Origasa H, et al: Effects of eicosapentaenoic acid on major coronary events in hypercholesterolaemic patients (JELIS): a randomised open-label, blinded endpoint analysis. Lancet 2007;369:1090-1098.

-Yuan JM, Ross RK, et al: Fish and shellfish consumption in relation to death from myocardial infarction among men in Shanghai, China. Am J Epidemiol 2001;154:809-816.

Yzebe D, Lievre M: Fish oils in the care of coronary heart disease patients: a meta-analysis of randomized controlled trials. Fundam Clin Pharmacol 2004;18:581-592. 